\title{
Smart Government
}

\section{Wie uns die intelligente Vernetzung zum Leitbild "Verwaltung 4.0" und einem smarten Regierungs- und Verwaltungshandeln führt}

\author{
Whitepaper
}

Version vom 14.09.2015

Prof. Dr. Jörn von Lucke

The Open Government Institute

Zeppelin Universität Friedrichshafen

Jörn von Lucke

Prof Dr

Lehrstuhl für Verwaltungs und Wirtschaftsinformatik

The Open Government Institute (TOGI)

Fon +497541 6009-1471

Fax +497541 6009-1499

joern.vonlucke@zu.de 


\section{Zusammenfassung}

The Open Government Institute (TOGI) der Zeppelin Universität in Friedrichshafen legt 2015 ein Whitepaper zu „Smart Government" vor, mit dem das Gestaltungspotential für Staat und Verwaltung im Internet der Dinge und im Internet der Dienste ausgelotet wird.

Ausgangspunkt ist die zunehmende intelligente Vernetzung realer und virtueller Objekte, mit der sich das Phänomen hinter dem Anglizismus „smart“ am besten umschreiben lässt. Mit Sensoren, Aktoren und Funkchips ausgestattete Dinge können miteinander und mit Menschen kommunizieren, über Apps und Dienste genutzt und in komplexere, so genannte cyberphysische Systeme (CPS) eingebettet werden. Systeme mit intelligent vernetzten realen und virtuellen Objekten werden so zu sich selbst steuernden Ökosystemen, die nicht nur bei Information und Analyse unterstützen, sondern auch Automation und Steuerung eigenständig übernehmen können.

Die deutsche Wirtschaft sieht darin eine echte Herausforderung für ihre Geschäftsmodelle und ihre Zukunft, denn diese kleine technische Erweiterung kann zu einer disruptiven Transformation führen und die Wettbewerbssituation etwa im Maschinenbau und Automobilbau grundlegend verändern. Als Reaktion darauf konkretisieren Wissenschaft, Wirtschaft und Bundesregierung mit der Initiative „Industrie 4.0“ das Leitbild der smarten Fabrik. Im öffentlichen Bereich wird das Leitbild der nachhaltigen smarten Stadt bemüht, um Projektvorhaben im Bildungs-, Energie-, Gesundheits- und Verkehrswesen aufzusetzen. Tatsächlich ist die öffentliche Verwaltung jedoch sehr viel breiter aufgestellt.

Um diese ganze Bandbreite abzubilden, wird „Smart Government" umfassend als die Abwicklung geschäftlicher Prozesse im Zusammenhang mit dem Regieren und Verwalten mit Hilfe von intelligent vernetzten Informations- und Kommunikationstechniken verstanden. Ein intelligent vernetztes Regierungs- und Verwaltungshandeln nutzt die Möglichkeiten intelligent vernetzter Objekte und CPS zur effizienten wie effektiven Erfüllung öffentlicher Aufgaben. Das Leitwort „Verwaltung 4.0" steht damit für die technische Integration von CPS in die öffentliche Verwaltung sowie für die Anwendung des Internets der Dinge und der Dienste, einschließlich der sich daraus ergebenden Konsequenzen für die Wertschöpfung, die Geschäftsmodelle sowie die nachgelagerten Dienstleistungen und die Arbeitsorganisation. Hieraus lässt sich ein verständliches wie visionäres Leitbild für eine intelligent vernetzte Verwaltung ableiten.

Ein solcher Ansatz muss integrierend angelegt sein. Im Kern geht es um die Frage, welcher smarten Objekte der öffentliche Sektor bedarf und in welche CPS diese einzubetten sind. Zugleich ist sicherzustellen, dass diese sich in das Internet der Systeme, das Internet der Menschen, das Internet der Daten, das Internet der Dinge und das Internet der Dienste einfügen. Hier ist eine besondere Sensibilität geboten, denn smarte Objekte und CPS können, auch unbeabsichtigt, gegen die freiheitlichdemokratische Grundordnung verstoßen. Information und Analyse, Automation und Kontrolle über das Internet der Dinge und das Internet der Dienste sind dort zu begrenzen, wo diese Schaden anrichten. In sechs Szenarien zu Smart Government werden erste Konkretisierungen zu Feuerwehr 4.0, Gericht 4.0, Finanzverwaltung 4.0, Standesamt 4.0, Landwirtschaft 4.0 und Bauverwaltung 4.0 vorgestellt. Mit ihnen soll der gemeinsame Dialog von Wissenschaft, Politik, Verwaltung, Wirtschaft und Zivilgesellschaft über die inhaltliche Gestaltung von Verwaltung 4.0 eröffnet werden. 


\section{Vorwort}

"Gehen Sie niemals mit Anglizismen in die deutsche Verwaltung, denn Sie fliegen hochkantig hinaus!"

Zugegeben haben es Professoren und Mitarbeiter vom The Open Government Institute aus Friedrichshafen schwer, weil bereits der Markenname nur aus englischen Ausdrücken bestehen. Im Kern ist die obige Botschaft aber zutreffend. Die deutsche Verwaltung wird sich niemals Konzepte überstülpen lassen, die für einen anderen Kulturkreis, eine andere Verwaltungskultur und einen anderen Rahmen entwickelt worden sind und deswegen selten passen. Das ist auch verständlich, denn kann kein Einfluss auf diese Konzepte mehr genommen werden, kann man sich nicht mit ihnen identifizieren. Damit fehlen die erforderliche Akzeptanz und jede konstruktive Weiterentwicklung. Dies wäre und ist auch nicht unsere Absicht.

Weltweit gibt es eine ganze Reihe an Trends, die von Technologien getrieben werden und gleich globale Auswirkungen haben. Da sich das Englische sehr weit verbreitet hat, überrascht es nicht, dass für diese Trends zuerst oft Anglizismen verwendet werden. Diese Trends erreichen auch Deutschland. Sie zu ignorieren wäre genauso unpassend wie sich mit ihnen nicht angemessen auseinander zu setzen, denn sie können bedeutende Potentiale zur nachhaltigen Verwaltungsmodernisierung bieten.

Mit „Smart Government" steht ein neues angelsächsisches Schlagwort vor der Tür, dem zahlreiche weitere Begriffe folgen werden. Uns geht es an dieser Stelle aus dem Blickwinkel von Wissenschaft und Forschung in erster Linie um das Phänomen hinter dem durchaus geschickt gewählten Begriff. Deswegen stellen wir uns auch gleich die Frage, wie wir in Deutschland ein „intelligent vernetztes Regierungs- und Verwaltungshandeln" mit Inhalten und Leben füllen wollen. So bleibt zwar die Wiedererkennbarkeit durch den Namen gesichert, das Konzept aber wird spezifisch für den deutschen Kontext entwickelt. Dabei stellt sich heraus, dass die Bundesrepublik Deutschland durch ihre Forschungsförderung zu "cyberphysischen Systemen“, „Industrie 4.0" und "smarten Fabriken" der Wirtschaft und der Wissenschaft bereits zu einer weltführenden Rolle verholfen hat. Dies sollte auch ein Ansporn für die Verwaltungsinformatik und die öffentliche Verwaltung sein.

Mit Definitionen, Leitbildern, Modellen, Szenarien und einer Forschungsagenda zu einem „intelligent vernetzten Regierungs- und Verwaltungshandeln“ und „Verwaltung 4.0“ soll mit diesem Whitepaper der gemeinsame Dialog von Wissenschaft, Politik, Verwaltung, Wirtschaft und Zivilgesellschaft über die Auswirkungen des Internet der Dinge und des Internet der Dienste auf Staat und Verwaltung eröffnet werden. Diese inhaltliche Auseinandersetzung, so das Zwischenfazit nach mehr als 18 Monaten interner und nicht immer einfacher Diskussion, wird keine leichte Angelegenheit werden und uns alle noch in den kommenden Jahrzehnten beschäftigen. Dennoch halten wir 2015 für den richtigen Zeitpunkt zum Einstieg. Noch besteht ein großes Gestaltungspotential, das wir bei gebotener Sensibilität auch im Sinne der Ingenieurwissenschaften und gestaltenden Wissenschaften (Design Sciences) konstruktiv nutzen sollten. Wir freuen uns auf Ihre Impulse!

Friedrichshafen, am 14. September 2015

Jörn von Lucke 


\section{Inhaltsverzeichnis}

1. Bestandsaufnahme: Smart bedeutet intelligente Vernetzung! ............................................. 1

1.1 Zunehmende intelligente Vernetzung ............................................................................. 1

1.2 Smarte Welt - Intelligent vernetzte Welt .................................................................... 2

1.3 Intelligent vernetztes Regierungs- und Verwaltungshandeln .............................................. 3

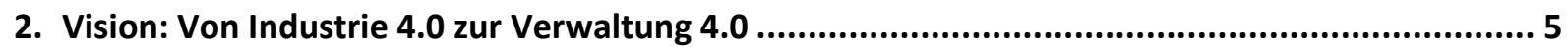

2.1 Industrie 4.0 und das Leitbild der smarten Fabrik ......................................................... 5

2.2 Smart Cities - Intelligente Lösungen für Bildung, Energie, Gesundheit und Verkehr ............. 6

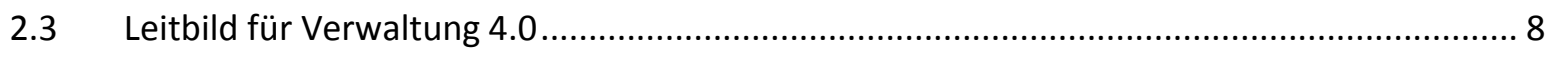

3. Integrierender Ansatz für einen intelligent vernetzten Staat ............................................... 11

3.1 Objekte mit Sensoren und Aktoren: Eingebettete Systeme .............................................. 12

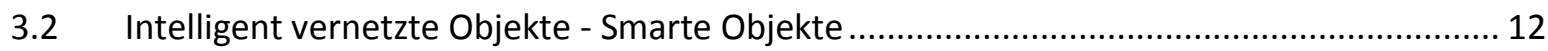

3.3 Mit cyberphysischen Systemen zu smarten Ökosystemen ................................................. 14

3.4 Internet der Systeme: Auf dem Weg zum intelligent vernetzten Verwaltungsnetzen? ....... 15

3.5 Internet der Menschen: Social Media und die öffentliche Verwaltung ................................ 17

3.6 Internet der Daten: Big \& Open Data und die öffentliche Verwaltung ............................... 17

3.7 Internet der Dinge und die öffentliche Verwaltung .......................................................... 18

3.8 Internet der Dienste und die öffentliche Verwaltung ..................................................... 19

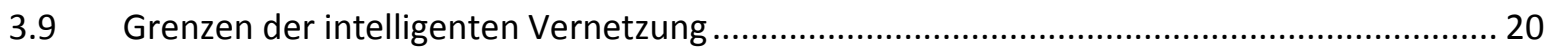

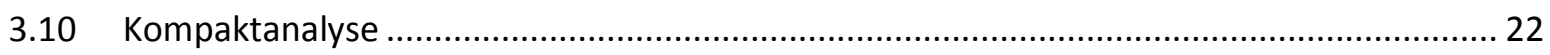

\section{Erste Szenarien zu Smart Government und Verwaltung 4.0:}

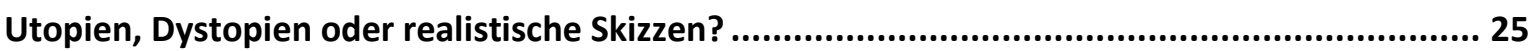

4.1 Feuerwehr 4.0 - Lösungsansätze für Freiwillige und Berufsfeuerwehr................................. 26

4.2 Gerichte 4.0 und Justizverwaltung 4.0 - Lösungsansätze für die Justiz.................................. 27

4.3 Finanzverwaltung 4.0 - Lösungsansätze für das Steuerwesen ............................................. 28

4.4 Standesamt 4.0 - Lösungsansätze für das Personenstandswesen ........................................ 29

4.5 Landwirtschaft 4.0 - Lösungsansätze für Landwirtschaftsverwaltung ….............................. 30

4.6 Bauverwaltung 4.0 - Lösungsansätze für Hochbau und Tiefbau ........................................... 31

5. Schritte auf dem Weg zur intelligent vernetzten Verwaltung 4.0 .......................................... 32

5.1 Forschungsagenda für "Smart Government" und „Verwaltung 4.0“ ..................................... 32

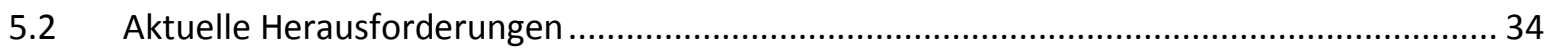

5.3 Warum Deutschland auf Smart Government nicht mehr länger warten sollte..................... 35

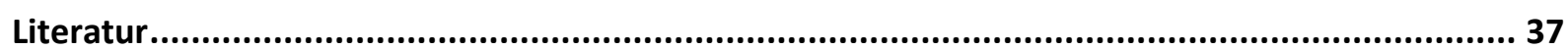




\section{Abkürzungsverzeichnis}

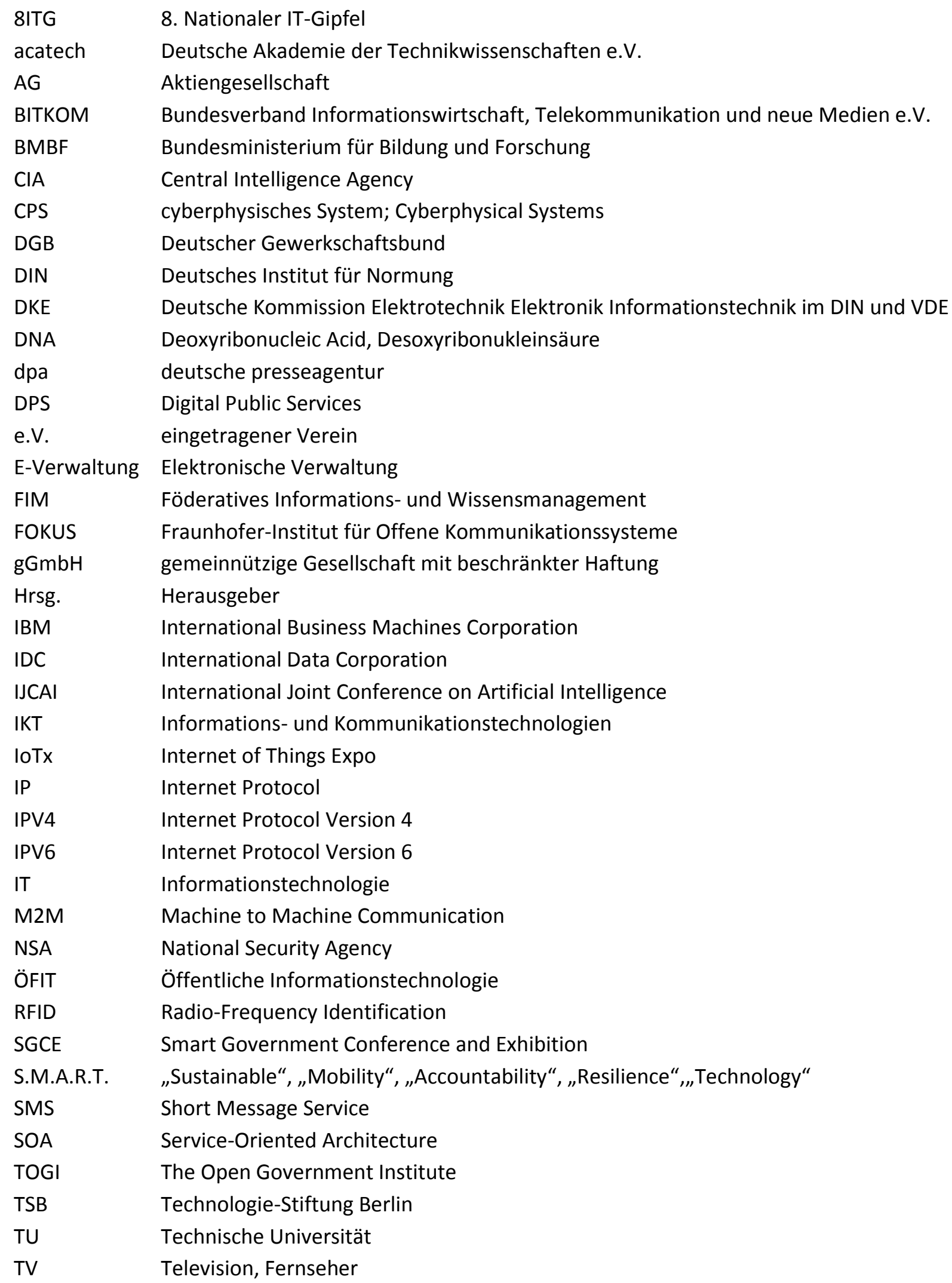


VDE

VDE-ITG

VDMA

WISE

WLAN

WSW

ZEW

ZVEI

Verband der Elektrotechnik Elektronik Informationstechnik e.V. Informationstechnische Gesellschaft im VDE Verband Deutscher Maschinen- und Anlagenbau Warsaw Institute for Economic Studies Wireless Local Area Network Wiener Stadtwerke Zentrum für Europäische Wirtschaftsforschung $\mathrm{GmbH}$ Zentralverband Elektrotechnik- und Elektronikindustrie e.V. 


\section{Bestandsaufnahme: Smart bedeutet intelligente Vernetzung!}

\subsection{Zunehmende intelligente Vernetzung}

Im Alltag lässt sich eine zunehmende intelligente Vernetzung beliebter Gegenstände feststellen. Mit jeder neuen Produktgeneration verfügen Endgeräte, oftmals ursprünglich analoge Dinge wie Zahnbürsten und Toaster, über erweiterte Funktionalitäten, bessere Sensoren, vielfältigere Reaktionsmöglichkeiten und eine breitbandige drahtlose Verbindung ins Internet. Ein geschicktes Marketing verkauft Telefone als "Smart Phones“, Tabletcomputer als "Smart Pads“, Fernseher als "Smart TV“ und Uhren als "Smart Watch“. Konsumenten freut dies, denn der Begriff "Smart" strahlt auch auf die Nutzer über und verleiht ihnen zusätzliche Gewitztheit und Cleverness. Und wer will schon gerne von sich behaupten, er sei das Gegenteil von „Smart“: Dumm?! Die intelligente Vernetzung von Objekten reicht mittlerweile jedoch sehr viel weiter und erfasst allmählich Autos und Lastkraftwagen, Schiffe, Flugzeuge, Maschinen und Fabriken. Prototypen von autonomen und sich selbststeuernden Automobilen werden längst auf Straßen getestet. Visionen von fahrerlosen Taxidroschken beflügeln Börsenkurse ausgewählter Unternehmen, sorgen aber auch für Demonstrationen und aufstandsähnliche Proteste.

Mit der zunehmenden intelligenten Vernetzung, mit der sich das Phänomen hinter "smart" am besten umschreiben lässt, müssen sich Wirtschaft und Gesellschaft, Wissenschaft und Feuilleton, Politik und Verwaltung zunehmend auseinander setzen. Dabei geht es nicht mehr nur um flächendeckendes Breitband, um neue Hardware und Software, um neue Apps und Informationssysteme. Es ist eine Vernetzung vielfältigster Objekte über Raum und Zeit zu beobachten, mit ganz neuartigen Kommunikationsfähigkeiten. Solche Objekte werden über das Internet ansprechbar und sind zugleich in der Lage, mit anderen virtuellen Objekten direkt zu kommunizieren. Diese kleine technische Erweiterung setzt ein gewaltiges revolutionäres Veränderungspotential frei, denn die Gestaltung smarter Objekte und der dazugehörigen Informationssysteme eröffnet ganz neue Möglichkeiten zur digitalen Transformation von Einrichtungen und Abläufen. Staat und Verwaltung werden sich aus diesem Grunde zunehmend intensiver mit dem Internet der Dinge und wegen der Vielzahl virtueller Objekte und Dienste auch mit dem Internet der Dienste auseinandersetzen müssen.

In diesem Whitepaper geht es um die zunehmende intelligente Vernetzung mit Blick auf das Regierungs- und Verwaltungshandeln. Ausgangsbasis ist die Fragestellung, was in einer "smarten Welt" unter "Smart Government" zu verstehen und mit welchen Inhalten ein „intelligent vernetztes Regierungs- und Verwaltungshandeln“ zu füllen ist (Kapitel 1). Mit Blick auf die deutsche Initiative Industrie 4.0 und das Leitbild einer smarten Fabrik stellt sich in Kapitel 2 die Frage, welche Schwerpunkte mit den Ansätzen smarter Städte bereits verfolgt werden und wie ein Leitbild für Verwaltung 4.0 als Ergänzung zu Industrie 4.0 aussehen könnte. Kapitel 3 setzt sich mit den technischen Rahmen einer intelligenten Vernetzung auseinander und integriert diese in einem gemeinsamen umfassenden Ansatz. Im vierten Kapitel werden beispielhaft sechs Szenarien vorgestellt, um sich möglichen Lösungsansätzen in verständlicher wie kompakter Form zu nähern. Zum Abschluss geht es in Kapitel 5 um die nächsten Schritte wie die Zusammenstellung einer Forschungsagenda, der Bewältigung aktueller Herausforderungen und den Aufruf, mit einer inhaltlichen Auseinandersetzung ernsthaft zu beginnen. 


\subsection{Smarte Welt - Intelligent vernetzte Welt}

Weltweit ist es gelungen, mit dem Anglizismus „smart“ einen mittlerweile anerkannten Begriff für die nächste Stufe der digitalen Vernetzung zu finden. Im Kern geht es um die intelligente Vernetzung bestehender Objekte und Netzwerke, die mit erweiterter Funktionalität in IT-Systeme eingebettet werden und dann eine virtuelle Identität erhalten, mit der kommuniziert werden kann. Im Deutschen steht das Adjektiv "smart" für "schnell, gewitzt und schlau“, "geschickt, durchtrieben, pfiffig und findig" sowie "elegant, hübsch und schneidig" (Wiktionary 2011-15). Das passende Antonym müsste eigentlich „dumm" lauten. Mit Blick auf die digitale Vernetzung wäre "unvernetzt" oder „auf sich allein gestellt" sehr viel passender. Auch wenn die Bezeichnung „S.M.A.R.T.“ den Anfangsbuchstaben der Begriffsfolge "Sustainable“, „Mobility“, „Accountability“, „Resilience" und „Technology" (Habbel 2014, S. 14) entspricht, geht es nicht bloß um den Einsatz nachhaltiger, mobiler, rechenschaftspflichtiger und widerstandsfähiger Technologien.

Bei intelligent vernetzten Objekten (engl.: Smart Objects) geht es um erweiterte Dinge des Alltags, die zusätzlich mit Sensoren, Aktoren und einer Kommunikationseinheit ausgestattet sind und die über eine virtuelle Repräsentation im Internet eine eindeutig ansprechbare Identität erhalten. Interagieren solche Objekte miteinander oder mit Menschen, so wird ihnen umgangssprachlich eine "gewisse Intelligenz" (Smartness) zugesprochen, selbst wenn Denkvermögen und Weisheit nicht vorliegen sollten. Bei Bedarf lassen sich diese Dinge in so genannte cyberphysische Systeme (CPS) einbetten, mit denen eine intelligente Vernetzung realer smarter Objekte gewährleistet werden kann. Hinter dem Begriff einer "smarten Welt" steht damit die Anwendung des Internets der Dinge und des Internet der Dienste auf die reale Welt. Diese umfasst Lösungsansätze mit intelligent vernetzten realen und virtuellen Objekten in sich selbst steuernden (smarten) Ökosystemen. Dies macht einen entscheidenden Unterschied zu den bisherigen Ansätzen aus: Smarte Objekte und CPS unterstützen nicht nur bei Information und Analyse. Sie können auch Automation und Steuerung eigenständig und vom Menschen unabhängig übernehmen. Auf die damit verbundenen technischen Ansätze und ihre Konsequenzen wird in Kapitel 3 detailliert eingegangen.

Intelligente Vernetzung sollte nicht mit vernetzter Intelligenz verwechselt werden. Bei intelligenter Vernetzung geht es darum, dass reale oder virtuelle Objekte über ein verteiltes Netzwerk miteinander kommunizieren, aktivierte Sensoren ausgewertet und Aktionen initiiert werden. Die dahinter stehende Logik in Netzwerken und Objekten ist in der Regel recht einfach gestrickt, ohne dass sie mit menschlicher Intelligenz mithalten wird können. Bei vernetzter Intelligenz stehen dagegen Menschen und deren Intelligenz im Mittelpunkt, die sich zur Erreichung gemeinsamer Ziele mit Hilfe von Rechnernetzwerken und Informationssystemen zusammenschließen und so vernetzen. Damit geht es um IT-gestützte Formen der Zusammenarbeit wie etwa das Crowdsourcing, ein offenes Wissensmanagement oder Ansätze offener gesellschaftlicher Innovation (von Lucke 2012; Raffl et al. 2014).

Auch eine Abgrenzung zur künstlichen Intelligenz erscheint notwendig. Forschern in diesem Fachgebiet der Informatik geht es darum, IT-Systeme in die Lage zu versetzen, dass sie sich intelligent (wie etwa ein Mensch) verhalten. Einfachere Ansätze fokussieren sich auf die Lösung bestimmter Aufgabenstellungen, etwa ein Spiel, eine Fragestellung oder eine Prognose. Komplexere Ansätze führen zu sich selbststeuernden Systemen wie etwa autonomen Drohnen oder selbstfahrenden Kraftfahrzeugen. Die bisher unerreichte Königsdisziplin wäre die Schaffung von Humanoiden, also von menschenähnlichen Robotern mit eigenem Bewusstsein, (über-)menschlichem Verstand, eigenständigem Verhalten und eigener Kreativität. Intelligente Vernetzung ist derzeit eher dem unteren 
Niveau der einfacheren Ansätze zuzuordnen. Dennoch muss damit gerechnet werden, dass es in naher Zukunft auch komplexe autonome IT-Systeme geben wird, die in einer intelligent vernetzten Umgebung eigenständig intelligente Entscheidungen ohne menschliche Einflussnahme treffen können und diese im Rahmen ihrer Möglichkeiten auch sofort umsetzen werden.

Smarte Ansätze sind zunächst für die IT-Industrie von besonderem Interesse, denn eine intelligente Vernetzung verspricht ihr ein neues Geschäftsfeld und zusätzliche Einnahmen. Bestehende Konzepte zur Vernetzung sowie zu Hard- und Software können erweitert, neue Geschäftsmodelle generiert werden. So überrascht es nicht, dass etwa die IBM 2008 eine Initiative Smarter Planet (Palmisano 2008; http://www.ibm.com/smarterplanet/us/en/) auflegte, um gemeinsam „mit Vordenkern“ in Wirtschaft, Politik und Zivilgesellschaft über das Potential smarter Systeme nachzudenken. Gerade die Wirtschaft erkennt aber, dass das Internet der Dinge und das Internet der Dienste echte Herausforderungen für ihre etablierten Geschäftsmodelle bedeuten, wegen möglicher disruptiver Veränderungen die Wettbewerbssituation grundlegend verändern und das eigene Überleben in Frage stellen. Bereits seit 2006 setzen sich in Deutschland Wissenschaft, Industrie und Politik mit den Auswirkungen smarter Fabriken, smarter Produkte, smarter Daten und smarter Dienste in einer „Smart Service Welt" auseinander (acatech 2011, Forschungsunion/acatech 2013, acatech 2015).

\subsection{Intelligent vernetztes Regierungs- und Verwaltungshandeln}

2009 entschloss sich das Emirat Dubai zur Errichtung des „Dubai Smart Government Department" (http://www.dsg.gov.ae), mit dem die Zuständigkeiten für das Gesamtangebot an Verwaltungsinformationen und elektronischen Verwaltungsleistungen neu gebündelt wurden. Bereits seit dem Jahr 2000 wurde im Smart Government Projekt mit smarten nachhaltigen Technologien für das städtische Umfeld experimentiert, die in vielen staatlichen Bauvorhaben eine Rolle spielen sollen. Mittlerweile wurden die E-Government-Aktivitäten des Emirats auf Smart Government hin neu ausgerichtet. Die Smart Government \& Smart Cities Conference (http://gccsmartgovernments.com) sowie der zusammen mit der Internet of Things Expo (IoTX: http://www.iotx.ae) stattfindende Smart Government Conference and Exhibition (SGCE: http://imconnected-tv.com/en) sollen den Wissenstransfer in die Region sichern und lokal entwickelte intelligent vernetzte Lösungen vermarkten.

Das Marktforschungs- und Beratungsunternehmen International Data Corporation (IDC) definiert "Smart Government" als die Implementierung eines Bündels an Geschäftsprozessen und darunter liegenden IT-Systemen, die sicherstellen, dass der Informationsfluss zwischen Behörden und Programmen nahtlos funktioniert, so dass die für die Bürger bestimmten Verwaltungsleistungen über alle Behörden und Aufgabenfelder hinweg intuitiv in hoher Qualität erbracht werden können (Rubel 2012, S. 2). Inhaltlich erschließt das dazugehörige „Smart Government Maturity Model“ (Rubel 2012, S. 10) aber eher E-Government und ein offenes Regierungs- und Verwaltungshandeln. 2014 versteht das Marktforschungsunternehmen Gartner unter "Smart Government" die Integration von Informations-, Kommunikations- und darunterliegenden operationalen Technologien, um bei Planung, Bewirtschaftung und Betrieb in verschiedensten Fachbereichen, Prozessketten und Zuständigkeiten nachhaltigen öffentlichen Mehrwert zu generieren (Gartner 2014). Unter den zehn dafür relevanten Technologietrends befindet sich bereits explizit das Internet der Dinge. Dass „Smart Government" aber weiterhin eher im klassischen Sinne als „geschicktes und gewitztes Regierungsund Verwaltungshandeln" verstanden wird, also eher intelligent der jeweiligen Situation angepasst, unterstreicht mustergültig der ehemalige US-Präsident Bill Clinton mit seinem 2011 erschienenen Buch „Back to Work: Why We Need Smart Government for a Strong Economy“ (Clinton 2011). 
In Deutschland fördert die Bundesregierung seit 2006 auf Wirtschaft und Industrie ausgerichtete Forschungsaktivitäten rund um das Internet der Dinge und das Internet der Dienste. Das auch Forschungsbedarf für ein intelligent vernetztes Regierungs- und Verwaltungshandeln besteht, wird erst seit 2015 sichtbar. Die auf die Sektoren Bildung, Energie, Gesundheit, Verkehr und Verwaltung ausgerichtete Initiative "Intelligente Vernetzung" (http://www.bmwi.de/DE/Themen/DigitaleWelt/initiative-intelligente-vernetzung.html sowie der Wettbewerb "Zukunftsstadt" (https://www.wettbewerb-zukunftsstadt.de) sollen gemeinsam mit den Bürgern vorbildhafte Lösungen zusammentragen, Leitbilder erarbeiten und Konzepte vorbereiten. Der Begriff "Smart Government" selbst hat bisher in Deutschland noch keinerlei Bedeutung erlangt, was auch an einer in Staat und Verwaltung verbreiteten Abneigung gegen Anglizismen liegen könnte. Jeder Staat, jede Regierung und ihre Verwaltungen werden sich aus unterschiedlichen Erwägungen heraus mit einem eigenen Definitionsansatz dem Konstrukt "Smart Government" nähern. Wie bei dem Schlagwort „Open Government" in Deutschland 2009-12 vorgeführt, kann es nicht darum gehen, woanders bewährte Konzepte direkt und unreflektiert zu übertragen. Vielmehr wurde damals ein eigenständiger Ansatz eines offenen Regierungs- und Verwaltungshandelns gesucht und erarbeitet. Übertragen auf das Schlagwort "Smart Government" bedeutet dies, dass ein gemeinsames Verständnis für ein intelligent vernetztes Regierungs- und Verwaltungshandeln auszuarbeiten ist, dass ein smartes Handeln wie smarte Technologien umschließt.

Mit diesem Beitrag soll das folgende umfassende Begriffsverständnis als die Häfler Definition von Smart Government (Friedrichshafener Definition; in Anlehnung an von Lucke/Reinermann 2000, S. 1) vorgeschlagen und in diesem Sinne auch weiter verwendet werden:

„Unter Smart Government soll die Abwicklung geschäftlicher Prozesse im Zusammenhang mit dem Regieren und Verwalten (Government) mit Hilfe von intelligent vernetzten Informations- und Kommunikationstechniken verstanden werden. Ein intelligent vernetztes Regierungs- und Verwaltungshandeln nutzt die Möglichkeiten intelligent vernetzter Objekte und cyberphysischer Systeme zur effizienten wie effektiven Erfüllung öffentlicher Aufgaben. Dies schließt das Leistungsportfolio von EGovernment und Open Government einschließlich Big Data und Open Data mit ein. Im Kern geht es um ein nachhaltiges Regierungs- und Verwaltungshandeln im Zeitalter des Internets der Dinge und des Internets der Dienste, die technisch auf dem Internet der Systeme, dem Internet der Menschen und dem Internet der Daten aufsetzen. Diese Definition umfasst sowohl die lokale oder kommunale Ebene, die regionale oder Landesebene, die nationale oder Bundesebene sowie die supranationale und globale Ebene. Eingeschlossen ist somit der gesamte öffentliche Sektor, bestehend aus Legislative, Exekutive und Jurisdiktion sowie öffentliche Unternehmen."

Mit diesem umfassenden Ansatz einer intelligent vernetzten Verwaltung, der technisch in Kapitel 3 genauer dargestellt wird, wird der zentrale Gedanke von Jimènez-Gomez et al. zu Smart Government aufgegriffen, demnach Open Government als Bestandteil von Smart Governance zu sehen ist und es kein Smart Government ohne Open Government geben kann (Jiménez-Gómez et al. 2015, S. 391). Übergeordnete Begriffe fordern zu weiteren Konkretisierungen auf. Insbesondere das Adjektiv „smart" mit seiner Bedeutungsvielfalt regt dazu an, sich über ein intelligent vernetztes Politisches Handeln (,Smart Politics“), eine intelligent vernetzte Gesetzgebung (,Smart Legislation“) und intelligent vernetzte Behörden („Smart Administration“), aber auch über smarte Bescheide, smarte Beamte, smarte Bürger und smarte Städte Gedanken zu machen. Benötigt werden konstruktive Leitvorstellungen zum Umgang mit dem Internet der Dinge und dem Internet der Dienste in Staat, Verwaltung und Gesellschaft, etwa vergleichbar wie die in Deutschland entwickelte Vision „Industrie 4.0“. 


\section{Vision: Von Industrie 4.0 zur Verwaltung 4.0}

\subsection{Industrie 4.0 und das Leitbild der smarten Fabrik}

Seit 2006 fördert die Bundesregierung im Rahmen ihrer Hightech-Initiative gezielt Forschung rund um das Internet der Dinge. Diese Aktivitäten mündeten rasch in Anwendungsszenarien für intelligente Objekte in industriellen Herstellungs- und Wertschöpfungsprozesse. Auf der Hannover Messe 2011 ist die Idee „Industrie 4.0“ erstmals der breiten Öffentlichkeit vorgestellt worden. Im gleichnamigen Zukunftsprojekt "Industrie 4.0" wurde ab November 2011 das Potential intelligenter Objekte, CPS und ihrer Vernetzung im industriellen Kontext vertieft. Im Oktober 2012 legte acatech erste Umsetzungsempfehlungen für das Zukunftsprojekt Industrie 4.0 vor (acatech 2012). Das Ziel lautet: „Deutschland läutet die vierte industrielle Revolution ein.“ Zur Hannover Messe 2013 stellte der Arbeitskreis „Industrie 4.0“ (Forschungsunion/acatech 2013) der Bundeskanzlerin seinen Abschlussbericht vor. Die Promotorengruppe Kommunikation der Forschungsunion Wirtschaft und acatech als Deutsche Akademie der Technikwissenschaften konkretisierten ihre Umsetzungsempfehlungen für das Zukunftsprojekt Industrie 4.0 und beleuchteten die Voraussetzungen für den erfolgreichen Aufbruch ins vierte industrielle Zeitalter. Im Oktober 2013 ergänzte das BMBF dies mit dem „Zukunftsbild Industrie 4.0“ als zur Diskussion gestellten Hypothese (BMBF 2013). Die Deutsche Kommission Elektrotechnik Elektronik Informationstechnik im DIN und VDE präsentierte im Dezember 2013 eine erste Normungs-Roadmap „Industrie 4.0" (DKE 2013). Seit 2014 arbeitet die von den Industrieverbänden BITKOM, VDMA und ZVEI neu gegründete und durch eine Geschäftsstelle unterstützte Plattform Industrie 4.0 (http://www.plattform-i40.de; Abbildung 1) die weiteren Umsetzungsschritte in unterschiedlichen Arbeitsgruppen aus.

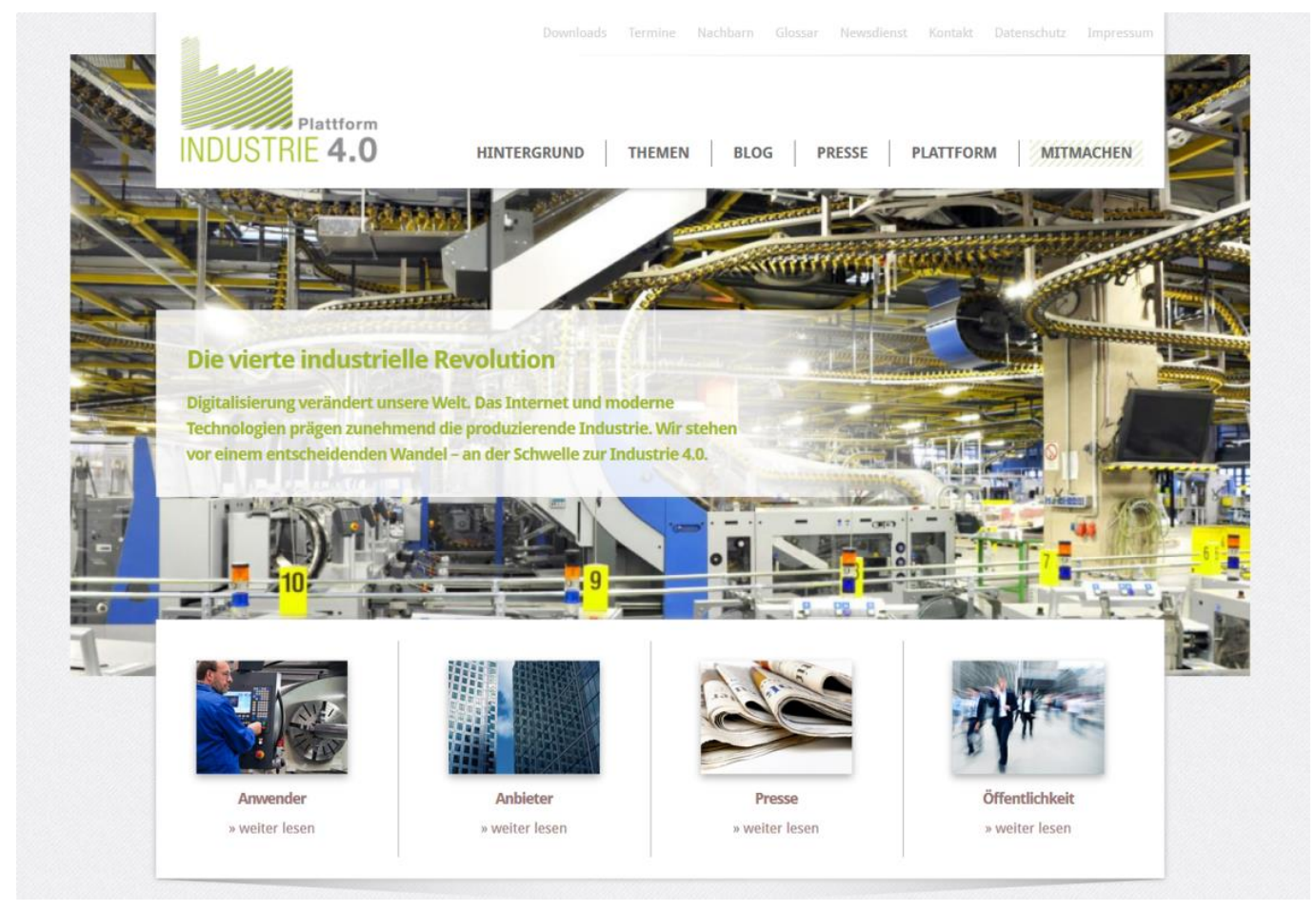

Abbildung 1: Plattform Industrie 4.0: http://www.plattform-i40.de

Quelle: Geschäftsstelle Plattform Industrie 4.0. 
Mit der Vision "Industrie 4.0" wird auf die Vernetzung, die Kommunikation und den Austausch von und zwischen intelligenten Objekten und sogenannten CPS gezielt, durch die sich die vierte industrielle Revolution (nach Einführung mechanischer Produktionsanlagen mit Dampfkraft, arbeitsteiliger Massenproduktion mit Elektrizität und Automation mit IT und Elektronik) erneut abheben wird: "Industrie 4.0 meint im Kern die technische Integration von CPS in die Produktion und die Logistik sowie die Anwendung des Internets der Dinge und Dienste in industriellen Prozessen - einschließlich der sich daraus ergebenden Konsequenzen für die Wertschöpfung, die Geschäftsmodelle sowie die nachgelagerten Dienstleistungen und die Arbeitsorganisation. "(Forschungsunion/acatech 2013, S. 18)

Die deutsche Industrie ist an einer raschen inhaltlichen Auseinandersetzung zu "Industrie 4.0" interessiert, weil sie negative Auswirkungen für die eigenen Unternehmen, ihre Zukunftsfähigkeit und damit den Standort Deutschland befürchtet, sollte dieses Forschungs- und Entwicklungsfeld nicht angemessen und zum Nutzen der Wirtschaft entwickelt werden.

Gerade der mit Industrie 4.0 verbundene grundlegende Paradigmenwechsel wird für disruptive Veränderungen sorgen: Hochautomatisiert lassen sich auch individualisierte Produkte mit einer Losgröße eins in smarten Fabriken fertigen. Massenindividualisierung wird so bei niedrigen Durchlaufzeiten ermöglicht. Intelligente Produkte unterstützen aktiv die Produktionsprozesse. Aufträge steuern sich zum Teil selbst durch dynamische Wertschöpfungsketten und -netzwerke. Autonome, sich selbst organisierende Produktionseinheiten ersetzen vorgeplant betriebene Produktionssysteme. Assistenzsysteme unterstützen in der Arbeit. Individuelle Verfügbarkeitskalender treten anstelle einer starren Mitarbeiteranwesenheit. Eine Ad-hoc-Vernetzung auf Produktions- und Geschäftsebene wird so erst möglich. All dies stärkt die dezentrale wie dynamische Selbstorganisation und kann zur Auflösung von klassischen Branchengrenzen und Verbänden führen (in Anlehnung an Pfirrmann 2013, S. 7, siehe aber auch Porter/Heppelmann 2014).

Die Forschungsunion und acatech empfehlen, dass Deutschland seine Stärken als „Fabrikausrüster der Welt" und bei eingebetteten Systemen nutzen sollte, um mit dem Einzug des Internets der Dinge und Dienste in der Fabrik eine neue, vierte Stufe der Industrialisierung einzuläuten. Dies würde nicht nur Deutschlands Produktivität und Wettbewerbsfähigkeit stärken, sondern auch zur Bewältigung anderer globaler und nationaler Herausforderungen beitragen (Forschungsunion/acatech 2013, 18).

\subsection{Smart Cities - Intelligente Lösungen für Bildung, Energie, Gesundheit und Verkehr}

Erste Ansätze zur Übertragung der Denkansätze von „Industrie 4.0“ auf urbane Räume führen rasch zum Konzept der "Smarten Städte“ (Smart Cities). Sehr treffend definiert wird dieser Begriff in einem auf nachhaltige Entwicklung ausgerichteten Bericht für die Wiener Stadtwerke:

"Die Smart City bezeichnet eine Stadt, in der systematisch Informations- und Kommunikationstechnologien sowie ressourcenschonende Technologien eingesetzt werden, um den Weg hin zu einer postfossilen Gesellschaft zu beschreiten, den Verbrauch von Ressourcen zu verringern, die Lebensqualität der BürgerInnen und die Wettbewerbsfähigkeit der ansässigen Wirtschaft dauerhaft zu erhöhen, mithin die Zukunftsfähigkeit der Stadt zu verbessern. Dabei werden mindestens die Bereiche Energie, Mobilität, Stadtplanung und Governance berücksichtigt. Elementares Kennzeichen von Smart City ist die Integration und Vernetzung dieser Bereiche, um die so erzielbaren ökologischen und sozialen 
Verbesserungspotenziale zu realisieren. Wesentlich sind dabei eine umfassende Integration sozialer Aspekte der Stadtgesellschaft sowie ein partizipativer Zugang." (WSW 2011, S. 19).

In seinem Buch "Die smarte Stadt" erschließt Kaczorowski die "smarten Handlungsfelder" Verwaltung \& Politik, Bildung, Wertschöpfung, Mobilität, Gesundheit \& Pflege sowie Energie \& Umwelt (Kaczorowski 2014, S. 67 ff.). Er beschränkt sich in seinen Ausführungen nicht nur auf intelligent vernetzte Objekte im „Internet aller Dinge“, sondern kombiniert in seinen Skizzen dies mit zahlreichen anderen Technologietrends für eine smarte Stadt: Breitband, Web 2.0, soziale Netzwerke, Cloud Computing, mobiles Internet, Big Data und vernetzte IT-Sicherheit (Kaczorowski 2014, S. 47 ff.).

Im technischen Kern geht es derzeit bei den meisten Smart City-Projekten um intelligent vernetzte und sich zum Teil selbst steuernde Lösungen für Bildung (Smarte Bildungsnetze: Smart Education Networks), für Energie (Smarte Energienetze: Smart Grid), für Gesundheit (Smarte Gesundheitsnetze für Telemedizin und Pflege: Personalisierte Medizin) sowie für Verkehr (Smarte Mobilitätsangebote: Individuelle Mobilität). In vielen Staaten handelt es sich hierbei um originäre Aufgabenfelder für Städte, Großstädte und Metropolen. Diese Felder haben eine herausgehobene wirtschaftliche wie gesellschaftspolitische Bedeutung. Sie versprechen Investoren attraktive Renditen. Sobald erste Prototypen überzeugen, wird zur Bewältigung der demographischen Herausforderung und der Energiewende in diesen Bereichen mit bedeutenden Investitionsentscheidungen zu rechnen sein. So bietet sich ein attraktiver Markt für smarte Ökosysteme, den derzeit zahlreiche Unternehmen mit eigenen strategischen Programmen erschließen. Zu erwähnen sind etwa Cisco (Smart+Connected Communities: http://www.cisco.com/web/strategy/smart connected communities.html), IBM (Smarter Cities: http://www.ibm.com/smarterplanet/us/en/smarter cities), Microsoft (City Next: http://www.microsoft.com/en-us/citynext), Oracle (Oracle's Smart City Platform Solution: http://www.oracle.com/us/industries/public-sector/national-local-government/city-platform) und die Fraunhofer-Gesellschaft (Morgenstadt: http://www.morgenstadt.de). Diese Erwartung rechtfertigt auch ein Engagement der zuständigen nationalen Ministerien und der Europäischen Union (http://ec.europa.eu/eip/smartcities). Bedauerlicherweise haben Bürger und ihre Wünsche dabei überwiegend noch eine untergeordnete Rolle inne. Mit dem Ansatz einer bürger-orientierten Smart City präsentiert Viktoria Beinrott einen umfassenden Ansatz um die Bürger, der auf einer partnerschaftlichen Zusammenarbeit aller Akteure basiert, konkrete Alltagsprobleme der Bürger löst und zunehmend auch strategische Beachtung bei der Umsetzung findet (Beinrott 2015).

Mit ihrem Leistungsportfolio ist die öffentliche Verwaltung insgesamt jedoch sehr viel breiter aufgestellt, als es die oben genannten Felder vermuten lassen (siehe etwa Weber 2015). Viele Bereiche des öffentlichen Sektors auf Bundes-, Landes- und kommunaler Ebene haben sich bisher noch gar nicht mit „Industrie 4.0“, „Smart Government" und den sich ihnen bietenden Möglichkeiten des Internet der Dinge und des Internet der Dienste auseinandergesetzt. Kruse und Hogrebe lieferten mit ihrer korrespondierenden Visionen „Verwaltung 4.0“ (Kruse/Hogrebe 2013) und „Deutschland 4.0" (Hogrebe/Kruse 2014) zwar Argumente für eine Standortentwicklung auf Basis der neuen Steuerungsmodelle und des E-Government. Jedoch fehlt auch bei ihnen ein wirklich eigenständiges Konzept zum Umgang mit den künftigen Herausforderungen, das auf smarten Objekten, CPS, dem Internet der Dinge und dem Internet der Dienste als zentralen Infrastrukturelementen aufsetzt. Kruse 2015 und Fromm 2015 zeigen in und mit ihren Beiträgen zudem, dass dem Leitgedanke „Verwaltung 4.0“ ernsthafte Gefahr droht, sich ohne inhaltliche Auseinandersetzung im "Bullshit-Bingo der Verwaltungsmodernisierung" zu verlieren. Die erforderliche ernsthafte Auseinandersetzung mit einer Vision und den dahinterstehenden Konzepten bleibt bisher noch weitgehend aus. 


\subsection{Leitbild für Verwaltung 4.0}

Eine Übertragung der Kerngedanken des Zukunftsprojekts Industrie 4.0 auf die öffentliche Verwaltung sollte das gesamte Angebots- und Leistungsportfolio des öffentlichen Sektors abdecken. Gerade die sich bietenden Gestaltungsoptionen von CPS, des Internet der Dinge und des Internet der Dienste eröffnen neuartige Möglichkeiten zur Modellierung und Gestaltung des künftigen öffentlichen Sektors. Daher gilt es ein Zukunftsbild „Verwaltung 4.0“ aufzustellen, welches sich mit den drei genannten Komponenten auseinandersetzt und auf zukünftige Anforderungen und Herausforderungen der Verwaltung im Rahmen eines Smart Government eingeht. In Anlehnung an die zentrale Definition von Industrie 4.0 (Forschungsunion/acatech 2013, S. 18) soll in diesem Beitrag für den öffentlichen Sektor das folgende Begriffsverständnis der Häfler Definition von Verwaltung 4.0 (von Lucke/Schumacher 2014, S. 10 und von Lucke/Schumacher 2015, S. 220) verwendet werden:

„Verwaltung 4.0 meint im Kern die technische Integration von cyberphysischen Systemen in die öffentliche Verwaltung sowie die Anwendung des Internets der Dinge und der Dienste im Rahmen der Prozesse des Regierens und Verwaltens - einschließlich der sich daraus ergebenden Konsequenzen für die Wertschöpfung, die Geschäftsmodelle sowie die nachgelagerten Dienstleistungen und die Arbeitsorganisation."

Ganz bewusst wird Bezug auf das Regierungs- und Verwaltungshandeln in seiner ganzen Breite und Vielfalt genommen. Wie die Speyerer Definition von E-Government (von Lucke/Reinermann 2000) umfasst auch dieser Ansatz sowohl die lokale oder kommunale Ebene, die regionale oder Landesebene, die nationale oder Bundesebene sowie die supranationale und globale Ebene. Eingeschlossen ist somit der gesamte öffentliche Sektor, bestehend aus Legislative, Exekutive und Jurisdiktion sowie öffentlichen Unternehmen.

Hieraus lässt sich ein verständliches Leitbild für Verwaltung 4.0 ableiten:

„Intelligente Objekte wie etwa smarte Brillen, smarte Fernseher, interaktive Leinwände und vernetzte Kleidungsstücke können in Ministerien, Behörden, Entscheidungsprozessen und Verfahrensabläufen sehr unterschiedliche Verwendung finden. Das gewaltigste Veränderungspotential liegt jedoch nicht im intelligenten Papier, sondern in dessen Überführung in ein intelligentes elektronisches Format. Die flächendeckende Einführung interoperabler elektronischer Akten- und Vorgangsbearbeitungssysteme verlagert Dokumente, Akten, Vorgänge und darauf aufsetzende Dienste in das Internet der Dinge und das Internet der Dienste. Zentrale Aufgaben der Informationsverarbeitung und Entscheidungsfindung lassen sich hochautomatisiert gestalten, ohne (dabei) menschliche Entscheidungsträger aus ihrer Verantwortung zu entlassen. Dies ermöglicht eine stärkere Massenbearbeitung von Einzelanträgen, Rechnungen und Genehmigungsprozessen. Intelligente Vorgänge unterstützen aktiv die Vorgangsbearbeitungsprozesse. Vorgänge steuern sich selbst durch Zuständigkeiten und dynamische Wertschöpfungsnetzwerke. Autonome, sich selbst organisierende Vorgangsbearbeitungssysteme mit Genehmigungsfiktion ersetzen die bewährte papierbasierte wie botenlastige Aktenhaltung. Portalbasierte einheitliche Ansprechpartner kümmern sich um das gesamte Anliegen der Bürger und Unternehmen, ohne diese mit administrativen Kenntnissen zu überfordern. Proaktive Verwaltungsleistungen und intelligente Bescheide ergänzen das Leistungsportfolio. All diese neuartigen kooperativen Ansätze stärken die dynamische Selbstorganisation und können zur Auflösung von klassischen Zuständigkeits- und Fachbereichsgrenzen führen." (von Lucke/Schumacher 2014, S. 10 und von Lucke/Schumacher 2015, S. 220-221). 
Gleichzeitig verändern sich durch den Null-Grenzkosten-Effekt (Rifkin 2014) die Kostenstrukturen durch die radikale Minimierung variabler Kosten. Lassen sich Aufgaben elektronisch schneller und kostengünstiger erledigen, entlastet dies auf lange Sicht die Haushaltskassen von Bund, Ländern und Kommunen.

Auf Grund der Vielfalt öffentlicher Aufgaben und denkbarer Eingriffsmöglichkeiten in Wirtschaft und Gesellschaft sollte sehr genau überlegt werden, wo Ministerien, Ämter und Verwaltungsmitarbeiter im Sinne von "Smart Government" mit welcher Form von Intelligenz auszustatten sind und wo zur Risikominimierung im öffentlichen Sektor bewusst Grenzen und Zuständigkeiten zu setzen sind. Schließlich ist durch den mit Verwaltung 4.0 verbundenen grundlegenden Paradigmenwechsel auch im Staat mit disruptiven Veränderungen zu rechnen, die in ihrer Breite derzeit noch nicht umfassend sichtbar sind und wegen ihrer Auswirkungen nicht überall auf positive Resonanz stoßen werden.

Beispielsweise kann ein „intelligent vernetztes Regierungs- und Verwaltungshandeln“ (Smart Government) von intelligent vernetzten Behörden (Smart Agencies) mit Hilfe eines intelligent vernetzten Verwaltungsnetzes (Smart Administrative Network) realisiert werden. Die Erfahrungen aus der Snowden-NSA-Abhöraffäre zeigen jedoch, dass sich diesbezügliche Vorstellungen von Nachrichtendiensten wie etwa der US-amerikanischen National Security Agency (NSA) oder einer Central Intelligence Agency $(\mathrm{CIA})$ in zentralen Punkten fundamental von der freiheitlich-demokratischen Grundordnung der Bundesrepublik Deutschland, etwa des Grundgesetzes und des Datenschutzes, sowie von den Vorstellungen vieler mündiger Bürger unterscheiden. In Deutschland wird nach dem Zweiten Weltkrieg durch Föderalismus, Gewaltenteilung, Ressortprinzip, Trennungsgrundsätze, kommunale Selbstverwaltung, Datenschutz, die informationelle Gewaltenteilung und das Recht auf informationelle Selbstbestimmung ganz bewusst sichergestellt, dass einzelne Behörden nicht zu viel wissen. Verwaltungsmitarbeiter in Deutschland sollen nicht in Erfahrung bringen dürfen, was der öffentliche Sektor insgesamt wissen könnte, wenn etwa alle vorhandenen Informationen mit Hilfe von Big Data, Smart Data und anderen Analyseansätzen zielgerichtet zusammengeführt und ausgewertet würden.

Konsequenterweise muss also für jede Fachverwaltung gesondert hinterfragt werden, inwiefern intelligent vernetzte Objekte in den Verfahrensabläufen sinnvoll sind und wie diese Lösungen konkret aussehen. Zeitnah sollten jene Bereiche bestimmt werden, in denen mit Unterstützung von CPS sehr viel intelligenter, effizienter und effektiver regiert, verwaltet, gehandelt, gestaltet und zusammengearbeitet werden kann. So können intelligente Objekte und Webdienste zur Verhaltensverfolgung, zur verbesserten Situationswahrnehmung und zur sensorgestützten Entscheidungsanalyse herangezogen werden. Auch Prozessoptimierungen und Ressourcenverbrauchsoptimierung sind vorstellbar. Unter Umständen wäre sogar eine Einführung von sich selbst steuernden komplexen autonomen Systemen sinnvoll und förderwürdig (Vgl. Chui/Löffler/Roberts 2010, S. $1 \mathrm{ff}$.). Bund, Länder und Kommunen sollten wissen, wo sie im zulässigen verfassungsrechtlichen Rahmen mit Ansätzen zu Verwaltung 4.0 effektiver ihre Aufgaben wahrnehmen, qualitativ hochwertige und zuverlässige Verwaltungsdienstleistungen anbieten, die Bürgerfreundlichkeit optimieren und ihren Mitarbeitern attraktive Arbeitsplätze anbieten können. Insofern wird es in den kommenden Jahren eine anspruchsvolle Aufgabe sein, zu jeder Fachaufgabe über zulässige intelligente Vernetzung und eine bewusste Grenzziehung nachzudenken, um den rechtsstaatlichen und datenschutzkonformen Rahmen einzuhalten. Schließlich geht es im Kontext des Leitgedankens "Verwaltung 4.0" um die inhaltliche Gestaltung des „smarten Staats" und der damit verbundenen digitalen Transformation des öffentlichen Sektors im 21. Jahrhundert im Sinne einer positiven Gesamtentwicklung. Konkrete auf öffentliche Aufgabenbereiche zugeschnittene Visionen bestimmter 
„smarter Behörden“ können dazu beitragen, die skizzierte Intelligenz greifbarerer und begrenzbarer zu machen, ohne eine versteckte Form eines Überwachungsstaats heraufzubeschwören. Selbstverständlich darf dabei der "smarte Bürger" nicht vergessen werden, dessen Kenntnisse, Erwartungen und Cleverness durch Vernetzung und unterstützende IT-Dienste ebenfalls weiter zunehmen werden.

Zur Gestaltung und Umsetzung von Smart Government und des Leitbildes Verwaltung 4.0 sollte ein echter Dialog gesucht werden. Nur im engen Austausch zwischen Legislative, Verwaltung, Wissenschaft, Wirtschaft und Zivilgesellschaft kann sichergestellt werden, dass auf breiter Legitimationsbasis die skizzierten Visionen zu Verwaltung 4.0 mit Inhalten gefüllt, davon Ziele abgeleitet, Umsetzungsstrategien bestimmt, Grenzen gezogen, Hindernisse erkannt und Herausforderungen konstruktiv überwunden werden. Die damit verbundenen rechtlichen, technischen, organisatorischen, administrativen, menschlichen, strategischen und politischen Fragestellungen sind im Rahmen eines ganzheitlichen Ansatzes schrittweise zu bearbeiten und beantworten. Im Sinne einer digitalen Transformation wird Verwaltung 4.0 zum Zusammenwachsen unterschiedlicher Ansätze und Konzepte beitragen können.

Aus diesen grundsätzlichen Überlegungen heraus wird verständlich, dass es fahrlässig wäre, Verwaltung 4.0 bloß als Begleitkonzept zu Industrie 4.0 aufzusetzen. Unbestritten muss die öffentliche Verwaltung auf Anforderungen von smarten Fabriken und smarten Produktionsprozessen angemessen reagieren können. Jedoch eröffnen sich durch intelligent vernetzte Objekte und Dienste für die Organisation und die Prozesse des Regierens und Verwaltens vollkommen neuartige Optionen, die unter den eigenständigen Dachmarken "Verwaltung 4.0" und "Smart Government" eine genauere Betrachtung und vor allem eine eigene Erschließung verdienen. 


\section{Integrierender Ansatz für einen intelligent vernetzten Staat}

Die bisherigen revolutionären Entwicklungssprünge in Staat und Verwaltung waren die Einführung der Kameralwissenschaft im absolutistischen Staat, der Bürokratie im 19. Jahrhundert und der informationstechnischen Systeme im öffentlichen Sektor im 20. Jahrhundert. Die nächste und vierte Stufe im 21. Jahrhundert wird von CPS im vernetzten öffentlichen Raum und den darin integrierten intelligent vernetzten Objekten geprägt. Ein smarter Staat, seine smarten Behörden und smarte Beamte werden deren Möglichkeiten im Rahmen der bestehenden Rechtsordnung nutzen. Aber auch smarte Bürger und smarte Unternehmen werden sich diesen Potentialen zur Einbringung und Durchsetzung ihrer eigenen Anliegen nicht verschließen wollen.

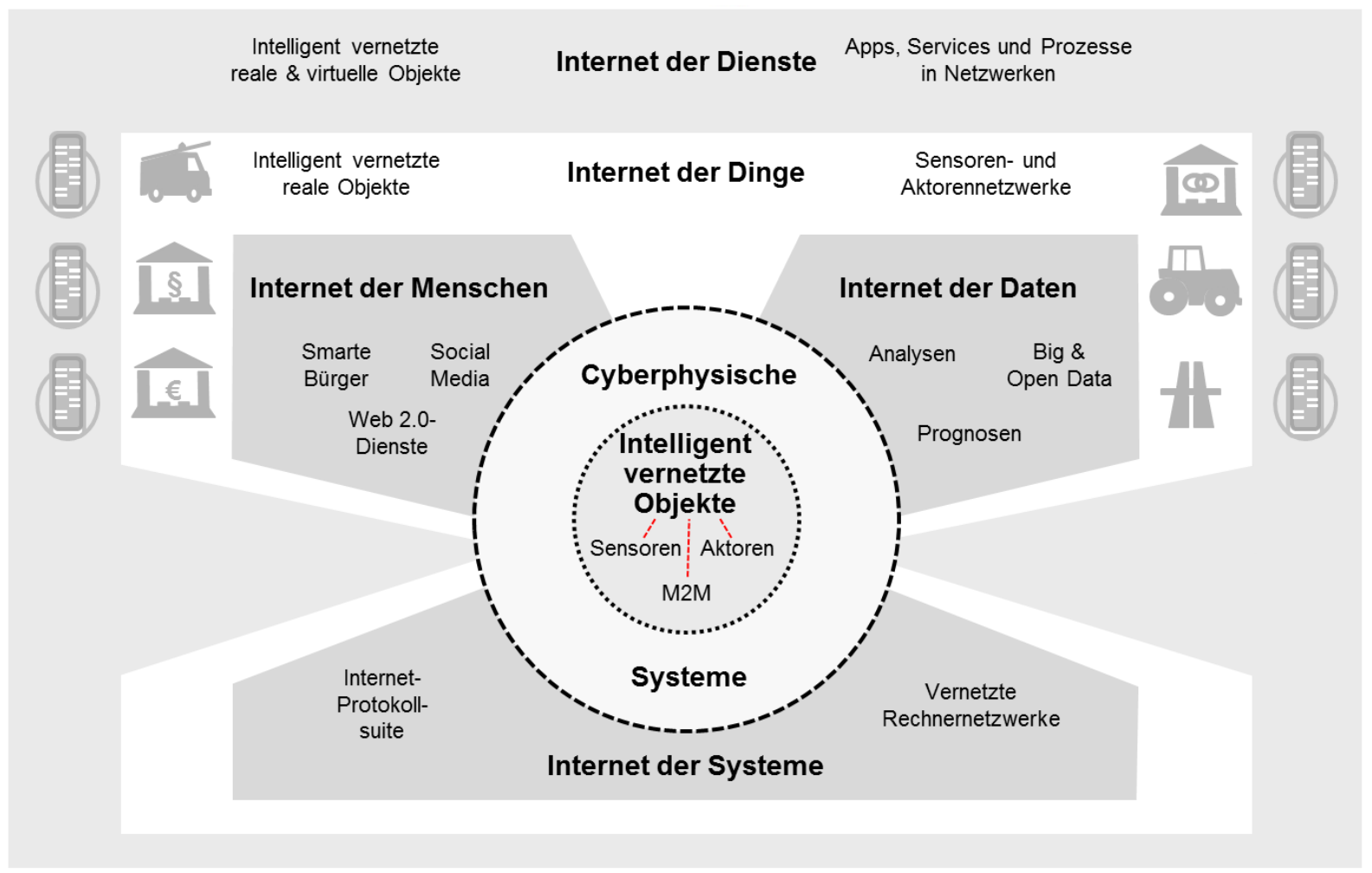

Abbildung 2: Integrierender Ansatz von Smart Government

Der Ansatz von Smart Government und ein in diesem Zusammenhang stehendes Leitbild "Verwaltung 4.0" (Abbildung 2) muss integrierend angelegt sein, um Investitionssicherheiten, Kompabilität und Zukunftsfähigkeit zu bieten. Im Kern geht es um die Frage, welcher intelligent vernetzten Objekte der öffentliche Sektor eigentlich bedarf (Abschnitt 3.1 und 3.2) und in welche CPS diese einzubetten sind (Abschnitt 3.3). Zugleich muss sichergestellt werden, dass diese nicht nur in einem abgeschlossenen Ökosystem funktionieren, sondern dass sie auch in das Internet der Systeme (Web 1.0: World Wide Web und Verwaltungsnetzwerke; Abschnitt 3.4), das Internet der Menschen (Web 2.0: Social Media; Abschnitt 3.5) und das Internet der Daten (Web 3.0: Semantic Web - Big and Open Data; Abschnitt 3.6) integrierbar sind. Essentiell ist auch die Gestaltung des Internet der Dinge mit seinen Sensor- und Aktornetzwerken (Abschnitt 3.7) sowie des Internet der Dienste (Abschnitt 3.8) mit seinen Web-Diensten (Web 4.0: Smarte Ökosysteme) aus staatlicher Sicht. Diese zentralen Kernelemente gilt es im Folgenden detailliert zu betrachten, um auf ihrer Basis verständliche Szenarien für den öffentlichen Sektor zu entwickeln. 


\subsection{Objekte mit Sensoren und Aktoren: Eingebettete Systeme}

Die eigentlichen revolutionären Veränderungen gehen von eingebetteten Systemen aus, also von Mikroprozessoren, die über Netzwerke miteinander verbunden sind und die über diese sogar miteinander kommunizieren können. Eingebettete Systeme sind Hardware- und Softwarekomponenten, die in ein Produkt integriert werden, um so weitere produktspezifische Funktionsmerkmale zu realisieren. Dadurch kann die Funktionalität und der Gebrauchswert des Produktes weiter erhöht werden (acatech 2011, S. 5 und Geisberger/Broy 2012, S. 254). Im Prinzip werden physische Objekte (Dinge) mit steuerbaren Chips ausgestattet und über Funk vernetzt. Durch eine virtuelle Repräsentation im Internet erhalten diese Objekte eine eindeutig ansprechbare digitale Identität. Über Sensortechnologien kann diese Funktionalität um die Erfassung von Zuständen (Temperatur, Feuchtigkeit, Schall, Licht, Bewegung, stoffliche Beschaffenheit) erweitert werden. Aktortechnologien ermöglichen zusätzlich auch die Ausführung von bestimmten Aktionen. Interagieren solche Objekte miteinander oder mit Menschen, so wird diesen Dingen umgangssprachlich eine "gewisse Intelligenz" zugesprochen. Gern spricht man von „intelligenten Objekten mit Sensoren und Aktoren“.

Sensoren sind technische Bauteile, die bestimmte physische oder chemische Eigenschaften qualitativ oder als Messgröße quantitativ erfassen und sie in eine digital weiterverarbeitbare Form umwandeln (Geisberger/Broy 2012, S. 139 und S. 255). Diese laufend generierten Daten können auf Nachfrage weitergeleitet werden. Ein Sensornetz ist ein System aus Sensorknoten, die entweder in einem infrastrukturbasierten oder einem sich selbst organisierenden Ad-hoc-Sensornetz zusammenarbeiten, um ihre Umgebung mittels Sensoren zu überwachen und die erfassten Daten, unter Umständen vorverarbeitet, weiterzuleiten (Geisberger/Broy 2012, S. 255). Sensoren unterschiedlichster Art befinden sich heute bereits in Smartphones, Tablets, Tischen, Videokameras, Geräten, Maschinen, Autos, Straßen, Häusern, Geschäften, Büros und Produktionsstraßen (Rifkin 2014b, S. 2). Technisch sind vielfältige Einsatzszenarien für Sensoren und ihre gebündelte Auswertung vorstellbar.

Aktoren sind Komponenten aus Software, Elektronik und Mechanik, die auf digitale Stellwerte reagieren und so regulierend in ein Regelungssystem eingreifen. Im Prinzip setzen sie von einem Steuerungscomputer ausgehende Befehle in mechanische Bewegung oder andere physische Größen wie Druck, Temperatur, Töne oder Licht um und beeinflussen so ihre Umgebung. Auch Aktoren gibt es in einer breiten Vielfalt. Bekannt sind vor allem mechanische Stellglieder in Regelkreisen, etwa Ventilsteuerungen, elektromechanische und hydraulische Antriebe sowie komplexe Steuerungssysteme, die sich um das koordinierte Zusammenspiel einer Vielzahl heterogener Aktoren in so genannten Aktornetzen kümmern (Geisberger/Broy 2012, S. 139 und S. 241). Auch Bildschirme, Lautsprecher, Drucker und 3D-Drucker sind in diesem Zusammenhang zu nennen.

\subsection{Intelligent vernetzte Objekte - Smarte Objekte}

Mittlerweile gibt es zahlreiche Objekte, die bereits über einen Funkchip, über einige Sensoren und über einzelne Aktoren verfügen. Ausgehend von einer ersten Bestandsaufnahme stellt sich die Frage, auf welche dieser smarten Objekte die öffentliche Verwaltung und ihre Mitarbeiter zu welchen Zeitpunkten zur öffentlichen Aufgabenerfüllung zugreifen könnten, sollten oder gar müssen.

Am Beispiel smarter Telefone (Smartphones) wird klar, dass diese zum Teil in unserer Gesellschaft schon eine sehr weite Verbreitung gefunden haben. Mit solchen Telefonen können Benutzer nicht nur telefonieren, sondern auch Gespräche und Mitteilungen aufzeichnen, Fotografien machen, 
Videofilme drehen, Kurznachrichten und E-Mails empfangen sowie digitale Objekte beliebiger Art versenden. Viele weitere Anwendungen sind als Apps herunterladbar. Automatisch generierte Nutzungsprotokolle lassen sich aus der Ferne und teils ohne Wissen der Eigentümer auswerten. So generierte Bewegungsprofile und Übersichten zum Kommunikationsverhalten sind für einige Behörden von großem Interesse, etwa zur Strafverfolgung. Die deutschen Datenschützer sehen dies seit Jahren mit Sorge vor einem Überwachungsstaat sehr kritisch. Auch Uhren, Armbänder, Fotokameras, Tablets und Laptops werden zunehmend intelligent vernetzt, um die so generierten Bewegungs- und Aktivitätsprofile auf Drittsystemen gezielt zu analysieren und zu bewerten. Diese für Freizeit-, Sport- und Gesundheitsanwendungen gedachten Datenprofile könnten durchaus auch für andere Zwecke verwendet werden, ohne dass dies ursprünglich so vorgesehen war.

Smarte Brillen erweitern das Blickfeld ihres Betrachters um digital visualisierte Zusatzinformationen. Damit verbessern sie seine Wahrnehmung der Realität (Augmented Reality). In Verbindung mit Spracherkennung, Gesichtserkennung, Videoaufzeichnung und Sprachsteuerung eröffnen sich so bisher ungeahnte Anwendungs- und Analysemöglichkeiten. Diese generieren nicht nur Mehrwerte und Freude, sondern stoßen aus vielfältigen ethischen Überlegungen und Sorge um die Privatsphäre auch auf viel Ablehnung (dpa 2015, S. 1).

Auch moderne Digitalfernseher lassen sich über WLAN-Verbindungen an das Internet anschließen. Ergänzend zum Zugriff auf das Fernseh- und Radioprogramm über Antenne, Kabel und Satellit kann so auf das Angebot der ergänzenden Mediatheken sowie weitere Zusatzinformationen zugegriffen werden. Allerdings können smarte Fernseher das Fernseh- und Internetverhalten ihrer Zuschauer protokollieren und transparent machen. Smarte und interaktive Whiteboards sind großflächige digitale Tafeln, die mit einem Computer verbunden sind und diesen über eine mit Sensoren ausgestatte Projektionsfläche steuern. Dies eröffnet vielfältige Ansätze für Visualisierung und haptische Steuerung auf einer großflächigen Projektionsfläche, was sich hervorragend als flexibles wie übersichtliches Cockpit eignet. Sobald allerdings Fernseher, Tablets, Laptops oder Whiteboards mit einer Videokamera und Mikrofon ausgestattet sind, besteht die Gefahr, dass Dritte von Außerhalb auf diese zugreifen und sich so ein Livebild der Nutzer aus der Kameraperspektive mit Optionen zur Aufzeichnung von Ton, Stand- und Bewegtbildern machen.

Diese Beispiele zeigen, dass smarte Objekte schon eine weite Verbreitung in unserem Alltag gefunden haben. Ihr Potential ist den Nutzern, der Wirtschaft, dem Gesetzgeber und der Verwaltung in der gesamten Vielfalt kaum voll bewusst. Aktuelle Forschungsschwerpunkte wie Drohnen, autonome selbstfahrende Autos, smarte Energiezähler, sich selbststeuernde energieeffiziente Wohnungen und smarte Häuser zeigen, dass man derzeit erst am Anfang der technischen Entwicklung steht und dass die Komplexität der Objekte weiter zunehmen wird. Zugleich erscheint es realistisch, dass einige Objekte durch intelligent vernetzte Steuerungseinheiten zu überschaubaren Kosten nach- und aufgerüstet werden können. So smart gemachte Objekte wie Fahrräder, Autos, Schiffe und Züge werden sich leicht über Befehle und Apps ganz neuartig nutzen, verfolgen und auswerten lassen.

Staat und Verwaltung müssen sich im Kontext von Smart Government zwei zentralen Fragen stellen. Einerseits geht es um die Frage der Regulierung. Wie soll mit vorhandenen intelligent vernetzten Objekten umgegangen werden, wenn deren Sensoren automatisch Nutzungs- und Bewegungsprotokolle generieren und deren Aktoren auch Schäden anrichten können. Freiwillige Selbstverpflichtungen der Anbieter, smarte Objekte datenschutzkonform (Privacy-by-default) zu gestalten und bei hoher Qualität IT-Sicherheit zu gewährleisten, überzeugen, solange kein Missbrauch erfolgt. 
Der Gesetzgeber wird jedoch dann Regulierungsbedarf sehen, wenn Schaden entsteht oder inakzeptable Handlungen beobachtet werden. Im Sinne einer Selbstverpflichtung gilt es in diesem Zusammenhang auch zu klären, für welche öffentlichen Aufgaben smarte Objekte eingesetzt werden sollten und wo Grenzen bei Datensammlung, Datenauswertung und darauf aufsetzenden Aktionen zu ziehen sind. Behörden sind verpflichtet, auf eine wirtschaftliche wie sparsame Aufgabenerledigung zu achten. Zugleich müssen sie die Bürger und Verbraucher vor unzulässigen staatlichen Eingriffen in die Privatsphäre, aber auch vor gezielten Manipulationen im Falle kriegerischer Angriffe schützen.

Zweitens stellt sich aus dem Blickwinkel der Verwaltungsinformatik die Gestaltungsfrage: Welche intelligent vernetzten Objekte benötigen Behörden eigentlich zu einer noch effizienteren Wahrnehmung öffentlicher Aufgaben? In diesem Zusammenhang sei etwa an Anforderungen an neuartige smarte Fußfesseln für den Justizvollzug, an smarte und auf Gefahr für Leib und Leben hinweisende Kleidungsstücke für die Feuerwehr, an smarte abhörsichere Telefone für den diplomatischen Dienst oder an smarte Prüfbrillen zur Abnahme von Bauvorhaben gedacht. Ideenwettbewerbe werden noch neuartigere Ansätze hervorbringen. Unternehmen müssen sich hier mit überzeugenden Vorschlägen und Realisierungen positionieren. Oft reicht dazu aber nicht nur eine gute Idee aus. Vielmehr wird auch eine hochwertige Umsetzung smarter Objekte in CPS erforderlich sein.

\subsection{Mit cyberphysischen Systemen zu smarten Ökosystemen}

Cyberphysische Systeme (CPS: Cyberphysical Systems) sind heterogen vernetzte Gebilde, die reale physische Objekte mit digitalen Informations- und Kommunikationssystemen verknüpfen und kombinieren. Es handelt sich um IT-Systeme als Teil von Geräten, Gebilden oder Prozessen, die über Sensoren unmittelbar physische Daten erfassen und durch Aktoren auf physische Vorgänge einwirken, die vor allem aber die erfassten Daten auswerten und speichern. Zudem können sie aktiv oder reaktiv mit der physischen und der digitalen Welt interagieren. Dazu sind sie über digitale Kommunikationseinrichtungen untereinander (M2M) und in globalen Netzen (Internet) verbunden, so dass sie die weltweit verfügbaren Daten und Dienste nutzen können (acatech 2011, S. 13 und Geisberger/Broy 2012, S. 22).

CPS greifen zur Aufgabenerledigung in der Regel auf eine Vielzahl intelligent vernetzter Objekte, eingebetteter Systeme oder Sensornetze zurück, die sie auch im großen Umfang und über räumliche Entfernung nutzen. Durch ihre Anbindung an das Internet können CPS eine Reihe von neuartigen Funktionen, Diensten und Eigenschaften anbieten. Leistungsstarke CPS werden ihre verteilte Anwendungs- und Umgebungssituation unmittelbar erfassen, zusammen mit den Nutzern diese interaktiv beeinflussen und ihr Verhalten im Hinblick so auf die jeweilige Situation gezielt steuern (Geisberger/Broy 2012, S. 22). Hieraus lassen sich smarte Ökosysteme entwickeln, in die IT-Systeme, Menschen, Daten, Dinge und Dienste gleichermaßen eingebunden werden und die sich teils selbst informieren, analysieren, überwachen und steuern. Ihre Vernetzung über das Internet bewirkt eine zunehmend nahtlose Verzahnung von der realen mit der digitalen Welt.

Acatech hat in seinen Studien das Potential und das Anwendungsspektrum von CPS in ersten ausgesuchten Bereichen bereits detailliert analysiert: Mobilität und Verkehr, Betreuung in der Medizin, intelligente Energienetze als Teile eines Smart Grids und intelligente Produktionsprozesse. Zudem werden Zug- und Flugverkehrssteuerungssysteme, Tsunami-Warnsysteme mit verteilter Erkennung, autonome Roboter und Drohnen bei gefährlichen Einsätzen sowie Sicherheits- 
überwachung und -unterstützung erwähnt (Geisberger/Broy 2012, S. 29 ff. und 67 ff.). CPS könnten in einer Fülle weitreichender Lösungs- und Anwendungsmöglichkeiten für alle Bereiche des Alltagslebens in bisher kaum vorstellbarer Weise Beiträge zu Sicherheit, Effizienz, Komfort und Gesundheit der Menschen leisten. Auf die Marktstrukturen wirken CPS aber hoch disruptiv, denn die Anbieter werden mit ihren neuartigen Ansätzen bestehende Geschäftsmodelle und die Wettbewerbssituation grundlegend verändern, klassische Branchen transformieren und bisherige Marktführer herausfordern (acatech 2011, S. 5).

Es ist eine große Herausforderung, vertrauenswürdige und verlässliche CPS für den öffentlichen Sektor zu konzipieren, zu bauen, zu vernetzen, zu steuern, zu kontrollieren und zu warten (acatech 2011, S. 5). Im Gegensatz zu Frühwarn- und Risikosystemen muss in Staat und Verwaltung im Prinzip sichergestellt werden, dass Menschen weiterhin Entscheidungen selbst treffen können, Automation und Assistenz des Systems also nur nutzen, ohne sich von Rechnern bevormunden zu lassen. Schließlich sollen intelligente Objekte, Maschinen und Systeme auch weiterhin den Menschen, dem Volk und seinem Wohle dienen, nicht aber zum Instrument der Überwachung, Kontrolle und Unterdrückung werden. Diese ersten grundsätzlichen Überlegungen verweisen auf disruptive Veränderungen, einen enormen Spannungsbogen, gewollte Grenzen und vorhandene Risiken, auf die bei der Konzeption von CPS im öffentlichen Sektor und ihrer Einbindung in das Internet der Systeme Rücksicht zu nehmen ist. Eine Einbindung der Bürger, der Politik, der Verwaltungsmitarbeiter und verschiedener Wissenschaften sollte daher von Anfang an ins Auge gefasst werden.

\subsection{Internet der Systeme: Auf dem Weg zum intelligent vernetzten Verwaltungsnetzen?}

Das auf Internet-Protokoll-Familie (IP-Suite) aufsetzende Internet bildet als virtuelles Rechnernetzwerk die technische Grundlage für die weitreichendste weltweite Vernetzung von Rechnern und anderen technischen Objekten. Auf Grund der Netzeffekte und der breiten globalen Verfügbarkeit ist eine Anbindung an das Internet für Nutzer und Organisationen hoch attraktiv. Schließlich können alle angebundenen Systeme auch miteinander kommunizieren. Allerdings wurde durch das unerwartet rasche Wachstum des Internet der durch 32 Bit auf $2^{32}$ Netzwerkelemente begrenzte Adressraum im IPv4 allmählich zu eng. Die Anzahl der Rechner und mobilen Systeme nimmt noch immer stetig zu. Mit dem IPv6 konnte nun ein 128 Bit Adressraum für $2^{128}$ Netzwerkelemente (rund 340 Sextillionen = $3,410^{38}$ Adressen) geschaffen werden. Damit besteht langfristige Investitions- und Zukunftssicherheit für diesen Netzwerkansatz. Die IP-Protokoll-Suite eignet sich somit uneingeschränkt für eine Vernetzung von Rechnern, intelligenten Objekten und CPS über den gesamten Globus.

Die IP-Familie setzt sich aus mehreren eigenständigen Protokollen zusammen, die alle miteinander zusammenarbeiten, harmonisieren und sich so gegenseitig ergänzen. Eine Vernetzung findet dabei auf unterschiedlichen Ebenen statt, auf der Anwendungsschicht, der Transportschicht, der Internetschicht und Netzzugangsschicht. Sie können verschiedenste Übertragungstechniken (Kupferkabel, Glasfaser, Richtfunk, Mobilfunk) und Anwendungen unterstützen. Die verschiedenen Protokolle sorgen dafür, dass mit intelligenten Lösungsansätzen der Datenaustausch immer effizienter funktioniert. Neue Protokolle können die IP-Familie auch weiterhin ergänzen und stärken.

Parallel zum Internet gibt es auch eine Reihe an geschlossenen Rechnernetzwerken, die entweder auf der IP-Suite oder auf anderen Protokollen aufsetzen. Eigenständige Netzwerke und virtuelle Privatnetzwerke machen überall dort Sinn, wo eine schnelle wie breitbandige Vernetzung zwischen 
Partnern sichergestellt werden muss, unbefugte Dritte keine Zugangsberechtigung erhalten sollen und zur Zusammenarbeit auf ein hohes Maß an Vertrauen, Datenschutz und Datensicherheit gesetzt wird. In Deutschland unterhalten Bund und Länder eine Reihe an eigenständigen Netzwerken und Verbindungsnetze zur Erfüllung öffentlicher Aufgaben, zumeist auf IP-Basis.

IP-Netzwerke können sehr unterschiedlich konfiguriert werden. Bei einfachen Netzwerken wird ganz im Sinne von Netzneutralität nur auf eine technische Übermittlung von Datenpaketen gesetzt, ohne das vielfältige Angebot netzbasierter Zusatzdienste und Dienstklassen zu berücksichtigen. Smarte Netzwerke lassen sich dagegen um vielfältige Server, Router und Dienste erweitern, so dass bereits während der Datenübertragung im IP-Netz mit einer Aufgabenerledigung aus dem Netzwerk heraus begonnen werden kann. Zwischen den beiden Polen „Einfaches Netzwerk = Dummes Netzwerk“ und „Smartes Netzwerk = Intelligentes Netzwerk" sind verschiedenste Kombinationen denkbar. Intelligente Vernetzung bedeutet, dass sich Anwendungen aus einem Baukasten von Diensten bedienen, eine Vielzahl von Datenquellen nutzen, stationäre und mobile Sensoren auslesen und Informationen zur Weiterverarbeitung bereitstellen. Erwartet werden dabei neben schnellen Bandbreiten zunehmend auch hohe Datenqualität und hohe Verfügbarkeit.

Im Rahmen des Nationalen IT-Gipfels hat sich die Arbeitsgruppe 2 „Digitale Infrastrukturen als Enabler für innovative Anwendungen" mit intelligenten Netzwerken in den Bereichen Bildung, Energie, Gesundheit, Verkehr und Verwaltung auseinandergesetzt und Anwendungsszenarien skizziert (8ITG 2014). Bedauerlicherweise beschränkte sich das von Deutscher Telekom AG und Bundeswirtschaftsministerium federführend entwickelte Zielbild „Intelligente Verwaltungsnetze 2020" (8ITG 2014, S. 205 ff.) zu sehr auf Rahmenbedingungen und zu wenig auf weiterführende Inhalte. Auf Potentiale einer Verwaltung 4.0 wurde nicht einmal eingegangen. Weitere Konzepte zum Internet der Energie (Micro Grid: Geisberger/Broy 2012, S. 48 ff.), zur smarten Gesundheitsversorgung (Smart Health: acatech 2011, S. 22 und Geisberger/Broy 2012, S. 41 ff.) und zur smarten Mobilität (acatech 2011, S. 21 und Geisberger/Broy 2012, S. 40) wurden im Zukunftsprojekt Industrie 4.0 erarbeitet. Durch optimale Vernetzung und dynamische Selbststeuerung sollen sie zur Steigerung von Lebensqualität und Standortqualität und zur Sicherung von Arbeitsplätzen beitragen.

Kritisch zu hinterfragen ist, ob intelligente Verwaltungsnetze in Deutschland überhaupt sinnvoll und notwendig sind. Sicherlich wäre ihre Existenz im Interesse der technischen Netzbetreiber, denn zusätzliche Intelligenz muss auch technisch installiert und dauerhaft betrieben werden. Dies erschließt innen zusätzliche Einnahmequellen und erhöht Markteintrittsbarrieren wie Abhängigkeiten, für die letztendlich die Verbraucher und Steuerzahler aufkommen müssen. Intelligenz in Netzwerken macht aber dort Sinn, wo selbststeuernd der Energieverbrauch minimiert und in Echtzeit auf Veränderungen reagiert werden kann. Andererseits kann Intelligenz im Netzwerk die Netzneutralität in Frage stellen, etwa wenn bei der Datenübertragung Prioritäten und Nachrangigkeiten bis hin zur Blockade definiert werden. Zugleich eröffnen sich so Fenster zur Inspektion der Inhalte, zur Zensur und zur gezielten Diskriminierung. Werden die an Knotenpunkten übertragenen Daten zudem auf Server Dritter kopiert, analysiert und ausgewertet, wäre ein Überwachungsstaat lebendig gewordene Realität. Dieses Szenario wäre nicht im Sinne der Gründerväter und -mütter der Bundesrepublik Deutschland.

Förderlich wären möglichst einfache wie offene und netzneutrale Infrastrukturlösungen, auf denen leistungsfähige Server, Dienste, Objekte und Sensoren aufgesetzt und diese intelligent miteinander vernetzt werden können. Für ausschließlich verwaltungsinterne Anwendungsfelder und zur ebenenübergreifenden Vernetzung eignen sich beispielsweise gemeinsame Verwaltungs- und Verbindungs- 
netze. Aber auch das Internet eröffnet Bund, Ländern und Kommunen unter Wahrung der rechtlichen und politischen Vorgaben (Föderalismus, Ressortprinzip, Kommunale Selbstverwaltung, Konnexitätsprinzip, Subsidiaritätsprinzip) viel Potential für neuartige vernetzte Lösungsansätze, die durch technische und strategische Offenheit an weiterem Wert und Entwicklungspotentialen gewinnen. Ein geregelter netzbasierter Austausch vielfältigster Geräte und Objekte ist also auf ganz unterschiedlichen Wegen und Plattformen möglich. Dazu sind aber leistungsfähige, sichere und schnelle Netzwerke erforderlich.

\subsection{Internet der Menschen: Social Media und die öffentliche Verwaltung}

Das Internet der Computersysteme wird durch das Internet der Menschen (Web 2.0: Social Media) erweitert. Menschen können dank der gesellschaftlichen Medien vielfältige Angebote und Dienste einfach nutzen, ohne Details über Handhabung, Funktionsweise, Programmierung und Schnittstellen kennen zu müssen. Das Angebot dieser zweiten Generation an Webdiensten ist sehr breit gefächert. Es umfasst unter anderem Text-, Bilder-, Musik-, Audio- und Videoplattformen, soziale Netzwerke, Kurznachrichten, Blogs, Wikis, Apps, Foren und Bewertungsgemeinschaften. Viele dieser sich selbst tragenden Angebote werden als Dienste aus der Cloud bereitgestellt. Ihren jeweiligen Betreibern liegt viel an einer laufenden Erweiterung, um deren Attraktivität, Nutzerzahl und gegebenenfalls Werbeumsatz zu steigern. Auch intelligent vernetzte Objekte wie etwa Webcams oder Digitalkameras lassen sich in einige dieser Angebote sinnvoll integrieren. Dieses Internet zum Mitmachen eröffnet Staat und Verwaltung vollkommen neuartige und bisher unvorstellbare Möglichkeiten für Transparenz, Mitwirkung und Zusammenarbeit (von Lucke 2010, Geiger et al. 2013, von Lucke 2015).

\subsection{Internet der Daten: Big \& Open Data und die öffentliche Verwaltung}

Das Internet der Daten (Web 3.0: Semantisches Web) vernetzt vorhandene Datenbestände und erschließt sie so für eine offene Weiternutzung durch Dritte. Durch eine Öffnung ihrer Daten (Open Data) und deren Vernetzung (Linked Open Data) bieten sich für Behörden, Unternehmen, Verbände und Vereine neuartige Perspektiven zur Integration, Analyse, Bewertung und Nutzung von großen wie vielfältigen Datenbeständen, die nach Möglichkeit in Echtzeit auszuwerten sind (Big Data). Gerade in diesen Bereichen besteht für den öffentlichen Sektor auf Basis seiner Datenbestände ein großes Potential zur Generierung von zusätzlichem Wirtschaftswachstum (TSB 2014 und WISE 2014).

Sensoren in intelligent vernetzten Objekten erzeugen laufend Daten. Diese smarten Daten können nicht nur objektintern verwendet, sondern über Funk auch versendet, in der Cloud gespeichert und von Dritten ausgewertet werden. In ihrer Gesamtheit füllen solche Sensornetzwerke laufend ein „riesiges Meer an vernetzten und zum Teil auch offenen Daten“, welches im Prinzip nur durch den gezielten Einsatz von Analyse- und Prognosemethoden sinnvoll genutzt werden kann.

Für Staat und Verwaltung gibt es in Deutschland durch den Datenschutz und das Recht auf informationelle Selbstbestimmung strikte Vorgaben, inwieweit personenbezogene Datenbestände überhaupt gesammelt, genutzt und systematisch ausgewertet werden dürfen. Datenvermeidung und Datensparsamkeit sind aus gutem Grund hoch gehaltene Maxime. Sobald intelligente Objekte wie etwa Smartphones mit Personen in Verbindung gebracht werden können, handelt es sich bei den generierten Sensordaten um personenbezogene Datenbestände. Dies schränkt die Möglichkeiten zur Auswertung ein, da zunächst eine Zustimmung der Betroffenen erforderlich ist, die auch jederzeit widerrufen werden kann. Selbiges gilt in Bezug auf Unternehmen und Einrichtungen für den Umgang 
mit schutzwürdigen Betriebs- und Geschäftsgeheimnissen. Werden gesammelte Sensordaten jedoch vollständig anonymisiert, so dass sie nicht mehr auf einzelne Personen oder Organisationen zurückzuführen sind, dann lassen sie sich für weitere Auswertungen verwenden. Darüber hinaus ist gesetzlich geregelt, wann Daten endgültig zu löschen sind, um einen Missbrauch durch unberechtigte Dritte zu verhindern. Die schutzwürdigen Interessen des Bürgers und der Unternehmen werden in Deutschland mit Verweis auf die eigene Geschichte sehr hoch wertgeschätzt.

\subsection{Internet der Dinge und die öffentliche Verwaltung}

Das Internet der Dinge steht bereits für die vierte Generation der Web-Technologien (Web 4.0: Internet der Dinge und der Dienste), deren Veränderungskraft aus der intelligenten Verknüpfung von eingebetteten Hard- und Softwaresystemen mit intelligenten Diensten aus der Cloud resultiert. Das Internet der Dinge verbindet intelligent vernetzte Objekte mit ihren Sensoren und Aktoren sowie die darauf aufsetzenden CPS miteinander über die IP-Protokoll-Suite. Eingebettete Alltagsgegenstände und CPS lassen sich von Personen, Programmen, Diensten und Datenpaketen über eine IP-Adresse eindeutig identifizieren, ansprechen, nutzen und gegebenenfalls auch steuern. Das Internet der Dinge steht damit für die globale „elektronische Vernetzung von Alltagsgegenständen“ (BMBF 2013) und den direkten gegenseitigen Informationsaustausch von Objekten ohne menschliche Eingriffe im Sinne einer echten Kommunikation von Maschine zu Maschine (M2M-Kommunikation).

Das Internet der Dinge eröffnet Organisationen neuartige Möglichkeiten sowohl zur Information und Analyse als auch zur Automation und Kontrolle. Werden bestehende Datenbestände von Objekten geöffnet und vernetzt, erweitern sich die Potentiale für eine breitere Informationsakquise und für bessere Analysen. Dies kann sich positiv auf Möglichkeiten zur Entscheidungsunterstützung auswirken. Beispielsweise lässt sich auf Basis von Sensordaten das Verhalten von Personen, Dingen, Diensten oder Daten weltweit verfolgen. Bewegungen von smarten Produkten und ihrer Interaktionen sind jederzeit auswertbar. Geschäftsmodelle können sich auf solche verhaltensbasierte Datensammlungen stützen. Hier ist nicht nur an vereinfachte Recherchen, Überwachung und Berichterstattung durch die Nutzer selbst zu denken. Auch für Dritte eröffnen sich auf anonymer oder personalisierter Basis neuartige Auswertungsmöglichkeiten. Eine schnelle wie umfassende Auswertung der verfügbaren Sensordaten verbessert zudem jede Umgebungs- und Situationswahrnehmung in Echtzeit. Die Aufmerksamkeit von Entscheidungsträgern kann durch eine leicht verständliche Aufbereitung und Visualisierung erhöht werden. Computerunterstützte detaillierte Untersuchungen und transparente Datenvisualisierung stärken außerdem sensorgetriebene Entscheidungsanalysen, die menschliche Entscheidungsträger in schwierigen Planungs- und Entscheidungssituationen zur eigenen Unterstützung heranziehen (Chui/Löffler/Roberts 2010, S. 3 ff.).

Viele Datensammlungen im Internet der Dinge können auch zur Automation und Kontrolle verwendet werden. Auf Basis aktueller Sensordaten und Nutzereingaben lassen sich etwa Prozesse in geschlossenen Systemen optimieren und über Aktoren und Feedback-Mechanismen auch selbst steuern. Dies eröffnet Einsparmöglichkeiten bei Verbrauch, Energiekosten und notwendigen menschlichen Eingriffen. Eine konsequente Umsetzung dieser Gedanken mit CPS sollte zu einem optimierten Ressourcenverbrauch innerhalb eines ganzen Netzwerks führen. Zu denken ist hier an eine möglichst hohe Ausbringung bei gegebenen Ressourcen (Ressourcenproduktivität) und an einen möglichst niedriger Ressourceneinsatz bei gegebener Produktionsmenge (Ressourceneffizienz). Konsequent weitergedacht führt dies zu komplexen autonomen Systemen, die in offenen Umgebungen mit 
großer Unsicherheit eingesetzt werden, in denen schnell sofortige und robuste Entscheidungen durch automatisierte Systeme erforderlich sind (Chui/Löffler/Roberts 2010, S. 6 ff.).

\subsection{Internet der Dienste und die öffentliche Verwaltung}

Ebenfalls zur vierten Generation der Web-Technologien gehört das Internet der Dienste, in dem Dienste und Funktionalitäten als feingranulare Softwarekomponenten abgebildet und von Providern auf Anforderung über das Internet zur Verfügung gestellt werden. Web Services, Cloud Computing und standardisierte Schnittstellen ermöglichen dies. Die einzelnen Software-Bausteine sind dank als webbasierte Dienstleistungen (Web Services) miteinander integrierbar. Organisationen können modular einzelne Software-Komponenten zu komplexen und dennoch flexiblen Lösungen im Sinne einer dienste-orientierten Architektur (SOA: Service-Oriented Architecture) orchestrieren. Über Cloud Computing wird sichergestellt, dass auch externe IT-Ressourcen über das Internet genutzt werden können. Cloud-basierte Entwicklungsplattformen helfen, internetfähige Dienstleistungen über Organisationsgrenzen hinweg zu entwickeln und anzubieten. Dienstplattformen bündeln Komplettangebote und Prozesse, so dass Einzelangebote nicht mehr separat gesucht, verglichen, zusammengestellt und verknüpft werden müssen. Da alle Anwendungen webbasiert bereitgestellt werden, können diese plattform- und endgeräteunabhängig genutzt werden. Das Internet entwickelt sich so zum Baukasten für IKT-Anwendungen, IKT-Infrastrukturen und IKT-Dienste. In Verbindung mit Web 2.0-Technologien verbessert sich das Innovationspotential, da dank Virtualisierung und Vernetzung auf Anregungen, Impulse und Beschwerden zur Dienstleistungserbringung rasch reagiert werden kann (Berlecon Research 2010, S. 9 \& S. 14 f. und Geisberger/Broy 2012, S. 247).

Die enge Verzahnung des Internet der Dienste mit dem Internet der Dinge beruht darauf, dass sich eine Reihe an realen Dingen bei mindestens gleichwertiger Funktionalität auch in webbasierte Dienste überführen und um ergänzende durchdachte Funktionen erweitern lassen. Anstelle der technischen Weiterentwicklung von Dingen zu intelligenten Objekten tritt dann gleich die Neuentwicklung leistungsfähiger Web Services mit evolutionären wie teils disruptiven Folgen. Vielfach ist der webbasierte Dienst deutlich effizienter und effektiver, so dass auf das Original und damit verbundene Medienbrüche komplett verzichtet werden könnte. Elektronische Akten- und Prozessunterstützungssysteme bieten gegenüber Papier und Akten etwa neuartige Möglichkeiten zur gleichzeitigen gemeinsamen Einsicht und Bearbeitung, zur Prozessoptimierung, zur Kostensenkung und zur Effizienzsteigerung. Gerade der von einer papierbasierten Informationsverarbeitung geprägte öffentliche Sektor möchte dieses Potential im Rahmen der elektronischen Verwaltungsarbeit (EVerwaltung) künftig stärker nutzen. Der bewusster Verzicht auf Papier als Original eröffnet zudem für Eintrittskarten, Fahrkarten, Ausweise, Bescheide, Urkunden und Rechnungen neuartige webbasierte digitale Möglichkeiten und darauf aufsetzende Geschäftsmodellinnovationen.

Das Konzept des Internet der Dienste gewinnt erst allmählich an Konturen. Staat und Verwaltung werden eigene Wege zum Aufbau einer dienste-orientierten Architektur (Government SOA) gehen. Eine koordinierte Zusammenarbeit von Gebietskörperschaften, Ministerien und Behörden wäre aus Sicht der Steuerzahler wünschenswert, um Parallelentwicklungen bei Diensten und Prozessen zu vermeiden. Mit dem Aufbau eines Föderativen Informations- und Wissensmanagements (FIM \& FIM Föderal) werden hierzu die ersten infrastrukturellen Grundlagen gelegt. 


\subsection{Grenzen der intelligenten Vernetzung}

Das Konzept des Internet der Dinge legt die Grundlage zur umfassenden Vernetzung von Gegenständen über die IP-Protokolle. Intelligent vernetzte Objekte einschließlich ihrer Sensoren und Aktoren werden ansprechbar und steuerbar. Das Internet der Dienste erweitert diesen Ansatz zusätzlich auf virtuelle Objekte. Ansätze eines smarten Regierungs- und Verwaltungshandeln werden das Internet und die "Intelligenz" von CPS zur Bewältigung von Herausforderungen in einer Art und Weise nutzen, die in Dimensionen, Rechenleistung und Geschwindigkeiten bisher so kaum vorstellbar waren. Diesen Potentialen werden sich Staat und öffentliche Verwaltung dauerhaft nicht verschließen. Trotzdem ist hier eine besondere Sensibilität geboten. Künftige, auf intelligent vernetzten Alltagsgegenständen aufsetzende öffentliche Informationssysteme sowie entscheidungsunterstützende Systeme smarter Behörden dürfen nicht gegen die freiheitlich-demokratische Grundordnung verstoßen. Information, Analyse, Automation und Kontrolle über das Internet der Dinge und das Internet der Dienste sind innerhalb von Staat und Verwaltung dort zu begrenzen, wo sie die gewollten Lebenswelten, Arbeitswelten und Freiräume von Bürgern, Unternehmen, Beamten, Polizisten, Richtern, Angeklagten und anderen Betroffenen unverhältnismäßig einschränken oder gar Schäden oder Schlimmeres anrichten können.

Insofern muss sich die jetzige Gesellschaft und nach ihr jede weitere Generation mit Blick auf den jeweiligen technischen Fortschritt regelmäßig die Frage stellen, in wieweit sie sich bei Regierungsund Verwaltungsaktivitäten erstens für Information und Analyse von intelligent vernetzten Objekten und CPS unterstützen lassen wollen. So bedarf es gesetzlicher Regelungen, ob bei gebotener Notwendigkeit zu jedem Bürger auf Basis verfügbarer smarter Objekte überhaupt ein Bewegungsund Verhaltensprofil erstellt werden darf. Im Falle einer Befürwortung muss es Vorgaben für Umfang und Dauer der erforderlichen Vorratsdatenspeicherung geben, etwa ob nur ein grober Überblick, ein detailliertes Profil oder eine umfassende Verhaltensverfolgung zulässig seien. Für eine offene und freie Gesellschaft wäre es inakzeptabel, wenn aus smarten Objekten oder CPS Instrumente zur Überwachung und Unterdrückung der Menschheit oder bestimmter Gruppen werden. Im Kontext einer verbesserten Situationswahrnehmung gilt zu klären, welche smarten Objekte in welchen Situation verwendet werden dürfen, damit nicht gegen geltende Datenschutzvorgaben verstoßen wird. In aller Konsequenz mag dies bedeuten, dass bestimmte intelligent vernetzte Objekte zur Situationswahrnehmung gar nicht oder nur eingeschränkt verwendet werden dürfen. Sensorgestützte Entscheidungsanalysen profitieren von der zunehmenden Rechengeschwindigkeit. Je besser und passender die Vorschläge werden, desto mehr Vertrauen werden die Nutzer aufbauen. In einer freien Gesellschaft wird sich der kritische und sich selbst reflektierende Mensch dennoch nicht das eigene Denken nehmen lassen wollen. Jede Bevormundung durch Rechner, die über eine unverbindliche Empfehlung hinausgeht, wird er ablehnen. Damit geht es, ohne dass es uns in der jetzigen Phase der Neugier und ersten Entdeckung smarter Möglichkeiten bewusst wird, um die Suche nach dem schmalen Grat, bis zu dem eine intelligente Vernetzung für Staat, Bürger, Wirtschaft und Gesellschaft akzeptabel ist. Wenn auch noch nicht auf einer Bergwanderkarte, so doch zumindest auf einer nach beiden Seiten hin offenen Skala von 0 bis 100 wird man sich diesem schmalen Grat zumindest visuell (Abbildung 3) annähern können. Dahinter steckt die Frage, in wieweit eine Generation in Zeiten von Smart Government bei Informationsverarbeitung und Analyse auf die Macht von Algorithmen setzen wird. 


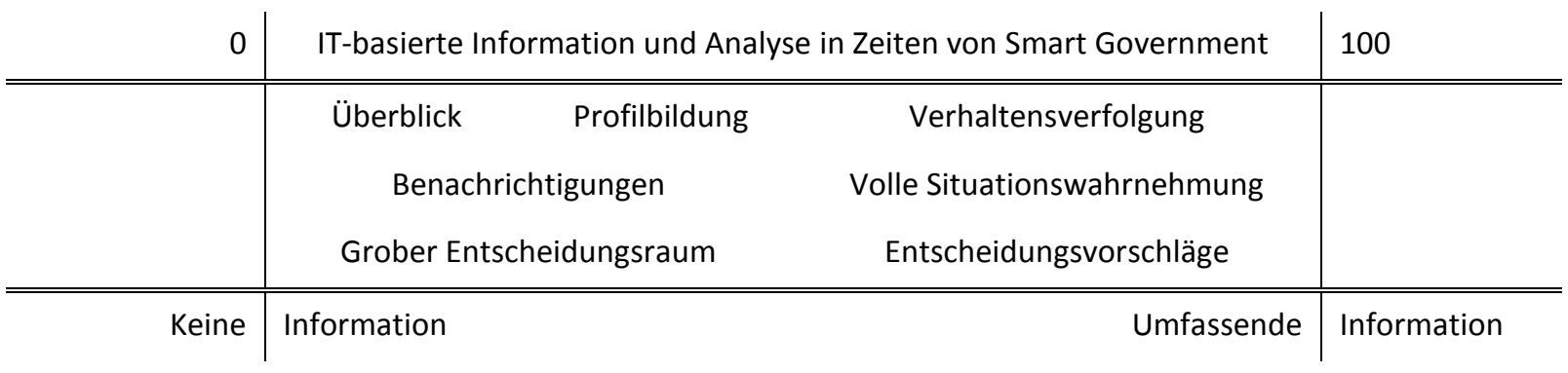

\section{Abbildung 3: Grenzen einer IT-basierten Information und Analyse in Zeiten von Smart Government}

Zweitens wird sich jede Generation auch mit der Frage auseinander setzen müssen, in wieweit intelligent vernetzte Objekte und CPS auch Steuerungs- und Kontrollaufgaben eigenständig übernehmen sollen. Sicherlich wird es zentrale Bereiche geben, etwa die Staatsführung, die Gesetzgebung oder die Rechtsprechung, in der die menschlichen Entscheidungsträger weiterhin voll in der Verantwortung bleiben wollen und automatische digitale Entscheidungssysteme prinzipiell ablehnen. Dennoch besteht innerhalb des öffentlichen Sektors eine breite Aufgabenvielfalt. So wird es Bereiche geben, in denen sich selbst steuernde autonome Systeme sinnvoll eingesetzt werden können. Zu denken ist an einfache Prozessoptimierungen, an eine Ressourcenverbrauchsminimierung bis hin zu komplexen autonomen Systemen. Wird bereits heute mit Verweis auf selbststeuernde Fahrzeuge die künftige Erfordernis von Taxifahrern in Frage gestellt (Ghosn 2015, S. 24), so muss auch der öffentliche Dienst in den kommenden Jahrzehnten damit rechnen, mit vergleichbaren Forderungen immer wieder konfrontiert zu werden. Auch hier eignet sich eine nach beiden Seiten hin offene Skala von 0 bis 100, um den schmalen Grat der Grenzziehung zwischen Akzeptanz und Ablehnung ITbasierter Steuerung und Kontrolle im Internet der Dinge und Internet der Dienste (Abbildung 4) zu verdeutlichen. Auch hier ist zu erwarten, dass jede Generation für sich selbst neu bestimmen wird, in wieweit sie eine Steuerung und Kontrolle durch Rechner akzeptieren wird, ohne sich bevormundet, überwacht oder unterdrückt zu fühlen. Nicht auszuschließen ist, dass künftige Generationen irgendwann die Entscheidungsgewalt über diese entscheidende Frage an Algorithmen verlieren könnten. Im Kontext eines smarten Regierungs- und Verwaltungshandeln wäre dies der Zeitpunkt, an dem Maschinen als menschliche Schöpfungen die Macht übernehmen würden und der Mensch längst an Bedeutung und Einfluss verloren hat. Insofern wird die gesellschaftliche Debatte über Akzeptanz und Ablehnung eines intelligent vernetzten Regierungs- und Verwaltungshandelns von Beginn an auch das Extremszenario einer vollständigen IT-basierten Automation und Steuerung mitberücksichtigen müssen.

\begin{tabular}{c|cc|c}
0 & \multicolumn{2}{|c|}{ IT-basierte Automation und Steuerung in Zeiten von Smart Government } & 100 \\
\hline \hline & Intelligente Systemkomponenten & Geschlossenes System & \\
& Manuell $\quad$ Automatik & Optimierter Ressourcenverbrauch & \\
& Einfaches autonomes System & Komplexes autonomes System & \\
\hline \hline Keine & Steuerung & Umfassende & Steuerung
\end{tabular}

Abbildung 4: Grenzen einer IT-basierten Steuerung und Kontrolle in Zeiten von Smart Government 
Ein in diesem Zusammenhang bedeutender Weckruf ist der offene Brief zu autonomen Waffen, mit dem die Internationale gemeinsame Konferenz zu künstlicher Intelligenz in Buenos Aires 2015 eröffnet wurde. Wissenschaftler und Meinungsmultiplikatoren fordern einen vorsichtigen Umgang mit den Errungenschaften und Möglichkeiten künstlicher Intelligenz. Sie sorgen sich vor einer weltweiten Aufrüstung mit autonomen Waffen, die dank künstlicher Intelligenz ohne menschliche Eingriffe Ziele auswählen und angreifen. Die Unterzeichner verlangen einen Bann offensiver autonomer Kampfsysteme, denn sobald diese selbstlernenden Roboter Steuerung und Kontrolle vollständig übernehmen werden, sind sie für die Menschheit nicht mehr zu kontrollieren, so dass es nahezu unmöglich wird, solche Systeme noch daran zu hindern, Menschen zu töten (IJCAI 2015).

\subsection{Kompaktanalyse}

Intelligent vernetzte Objekte, CPS, das Internet der Dinge und das Internet der Dienste sind neue Konzepte der angewandten Informatik, die zwar auf bestehenden technischen Errungenschaften aufsetzen, in ihren Auswirkungen aber Politik, Verwaltung, Wirtschaft und Gesellschaft erfassen und diese substantiell verändern werden. Viele Objekte des Alltags lassen sich mit Hilfe von ansprechbaren Prozessoren, Sensoren und Aktoren erweitern und neu gestalten. Die meisten papierbasierten Abläufe können über elektronische Akten- und Vorgangsbearbeitungssysteme sehr viel effizienter aufgesetzt und abgewickelt werden. Aus dem Blickwinkel der Verwaltungsinformatik wie der Wirtschaftsinformatik sind die damit zusammenhängenden Möglichkeiten bisher jedoch weder systematisch erfasst noch umfassend erschlossen worden. Obwohl im Kontext von "Smart Cities" bereits über Intelligenz in Energie-, Gesundheits-, Verkehrs- und Bildungsnetzen nachgedacht (8ITG 2014 und Geisberger/Broy 2012) wird, ist ein konkreter Anwendungsbezug zu den Kernbereichen der öffentlichen Verwaltung in Deutschland bisher noch nicht hergestellt und publiziert worden. Dieses Whitepaper wird im folgenden Kapitel 4 erste Szenarien für Verwaltung 4.0 präsentieren.

Häfler Definition, Kernelemente und die im Anschluss folgenden Skizzen haben den Zweck, ganz im Sinne einer wissenschaftlich-normativen gestaltungsorientierten Herangehensweise in der Öffentlichkeit ein Bewusstsein für die anstehenden Veränderungen durch Smart Government und Verwaltung 4.0 zu schaffen. Schließlich wird sich im globalen Kontext diese fortschreitende technische Entwicklung kaum aufhalten lassen. Folglich kann es nur noch darum gehen, wann, in welchen Bereichen, in welcher Form und in welchen Ausmaßen CPS den öffentlichen Sektor verändern werden. Im Interesse einer positiven Gesamtentwicklung sollten Stärken und Schwächen, Chancen und Risiken (Tabelle 1) miteinander abgewogen werden, um geeignete Schwerpunkte im öffentlichen Sektor auszuwählen, in denen in Pilotprojekten Potentiale, Nutzen, Herausforderungen und Grenzen erkundet und Lösungen gestaltet werden, um als Gesellschaft daraus zu lernen und zu profitieren. 


\section{Stärken}

- Integrierender Ansatz auf IP-Basis

- Intensivierung der Vernetzung

- Leitbild: Smarte Behörden

- Leitbild: Smartes Verwaltungshandeln

- Leitbild: Smarte Bürger

\section{Chancen}

- Innovationspotential und Impulse

- Neuartige intelligent vernetzte Dinge

- Neuartige intelligent vernetzte Dienste

- Neuartige cyberphysische Systeme

- Effizienzsteigerungen und Effektivität

- Kosten- und Gebührensenkungen
Schwächen

- Entwicklungsaufwand und -zeit

- Erforderliche finanzielle Ausgaben

- Unzureichende wissenschaftliche Grundlagen

- Forschungs- und Entwicklungskapazitäten

- Unzureichende politische Priorisierung

Risiken

- Kreativität, fehlende Gestaltungsbereitschaft

- Ungewissheit einer erfolgreichen Umsetzung

- Disruptiver Charakter der Veränderungen

- Fehlende dauerhafte Finanzierung

- Fehlende Akzeptanz und Mitwirkung

- Ängste durch Zerrbild "Gläserner Bürger“

\section{Tabelle 1: Kompaktanalyse von Smart Government}

IP-basierte E-Government-Lösungen haben dank des Internet weltweit Verbreitung gefunden. Einer funktionalen Erweiterung durch die Möglichkeiten des Internet der Menschen, des Internet der Daten, des Internet der Dinge und des Internet der Dienste und einer damit einhergehenden intensiveren Vernetzung von Systemen, Menschen, Daten, Dingen und Diensten werden sich Staat und Verwaltung daher kaum verweigern können und auch gar nicht wollen. Staaten werden es vielmehr als Stärke sehen, wenn sie im Rahmen von Verwaltung 4.0 eigene Leitbilder zu "smarten Behörden“, einem „smarten Regierungs- und Verwaltungshandeln“ und „smarten Bürgern“ entwickeln, um Gesellschaft, Wirtschaft und Verwaltung durch diesen Wandel erfolgreich zu führen. Solche Visionen geben Orientierung sowie Raum für Debatten, ethische Grenzziehungen, Ziele, Umsetzungstrategien und konkrete Maßnahmen, aus denen man insgesamt gestärkt heraustritt. Allerdings liegen derzeit noch keine umfassenden Lösungskonzepte für Verwaltung 4.0 oder gar in detaillierterer Form für eine Fachverwaltung 4.0 vor, welche intelligent vernetzten Objekte und CPS konkret für eine effektivere Aufgabenerfüllung einzuführen sind. Ihre Skizzierung, Planung, Entwicklung und Einführung werden noch mit erheblichen zeitlichen, personalintensiven und finanziellen Entwicklungsaufwendungen verbunden sein. Die wissenschaftliche Auseinandersetzung mit diesem neuen Forschungsfeld beginnt erst allmählich. Politik und Verwaltung sehen sich zudem gefordert, möglichst rasch einen Rahmen für eine sichere und vertrauenswürdige Informationsinfrastruktur zu schaffen, in der fremde Nachrichtendienste, Verbrecher und feindliche Armeen keine Chancen erhalten dürfen. In Zeiten knapper Budget- und Personalressourcen sind dies wichtige Punkte, welche vor einer angemessenen Auseinandersetzung mit Verwaltung 4.0 und diesbezüglichen Reformen zurückschrecken lassen könnten.

Der Weg zur Verwaltung 4.0 ist zudem mit einigen Risiken verbunden, die eine erfolgreiche Umsetzung durchaus ungewiss machen. Bei aller Veränderungsbereitschaft sind auch Kreativität und Gestaltungswille begrenzte Ressourcen. Der Stand der Wissenschaften, technische Grenzen wie Netzabdeckung, Bandbreiten und Standardisierung, die Verfügbarkeit kompetenter Vordenker und Finanzierungsengpässe werden kurz-, mittel- und langfristige Grenzen setzen. Jede Umsetzung von fachbereichsspezifisch erarbeiteten Verwaltung 4.0-Konzepten muss sich zudem mit den typischen rechtlichen, technischen, organisatorischen, finanziellen, strategischen und politischen Herausforderungen auseinandersetzen. Hinzu kommen offen artikulierte Bedenken und Akzeptanzschwierig- 
keiten aller Betroffenen aus Sorge vor Risiken und disruptiven Veränderungen. Gewerkschaften und Parteien werden sich zu Wort melden, um die Interessen der Beamten, Arbeitnehmer und Bürger angemessen einzubringen und durchzusetzen. Es ist damit zu rechnen, dass auch das Zerrbild des „gläsernen Bürgers“ benutzt wird, um gezielt mit Ängsten die eigene Verhandlungsposition zu verbessern. Insofern ist für eine erfolgreiche Umsetzung von Verwaltung 4.0 frühzeitig auch ein umfassendes Veränderungsmanagement gefordert.

Dennoch liegen gerade in den Innovationspotentialen und Wachstumsimpulsen die größten Chancen von Verwaltung 4.0. Schließlich lassen sich vollkommen neuartige intelligente Dinge und Dienste, insbesondere aber CPS für den öffentlichen Sektor konzipieren, mit denen sich öffentliche Aufgaben besser wahrnehmen lassen. So sind Verwaltung, Wissenschaft und Wirtschaft, also ingenieurwissenschaftlicher Geist und verwaltungswissenschaftliche Kenntnisse, gleichermaßen gefragt, wenn es um die Gestaltung intelligenter Objekte, Prozesse und Dienste, ihre Vernetzung und intelligente Steuerung in den jeweiligen Fachbereichen geht. Politisch vorgegebene Ziele wie Rechtsstaatlichkeit, Effizienzsteigerungen, Effektivität, individuelle Leistungsangebote, Mitarbeiterentlastung, Kostensenkungen sowie Aufgaben- und Ausgabenkontrolle müssen dabei berücksichtigt werden. Bürgern und Unternehmen kann so ein verbessertes Leistungsangebot zur Verfügung gestellt werden, das durch eine weitere Beschleunigung, niedrigere Gebühren, Individualität und Verlässlichkeit gekennzeichnet wird. Die assistierenden Funktionen der intelligenten Systeme in der Verwaltung 4.0 tragen zugleich zur Entlastung der Verwaltungsmitarbeiter bei. 


\section{Erste Szenarien zu Smart Government und Verwaltung 4.0: Utopien, Dystopien oder realistische Skizzen?}

Intelligent vernetzte Objekte wie Smartphones, smarte Brillen, Drohnen und vernetzte Autos finden bereits zunehmende Verbreitung. Mit ihnen lassen sich CPS im öffentlichen Raum aufbauen, in die Behörden, Unternehmen und Bevölkerung gleichermaßen eingebunden werden können. Ein öffentliches Tsunamifrühwarnsystem mit intelligent vernetzten Bojen im Meer und automatischen SMSWarnmeldungen ist ein erster überzeugender Ansatz für Effizienz und Effektivität. Gerade aus der Vernetzung von Systemen, Menschen, Daten, Objekten und Diensten im Internet der Dinge und im Internet der Dienste eröffnen sich neuartige Ansätze für Produkte, Dienste, Systeme und Geschäftsmodellinnovationen. Eine Verwaltung 4.0 profitiert von den sensorgenerierten, aber datenschutzkonform erhobenen Datensammlungen und einer raschen Handlungsfähigkeit durch autonome Systeme. Wertschöpfungsketten entwickeln sich so zu Netzwerken und Ökosystemen. Bestimmte öffentliche Aufgaben werden sich mit Smart Government viel effizienter und effektiver als bisher erfüllen lassen, was mit Einsparungen und einer Begrenzung der Risiken verbunden werden könnte.

Die konkreten Potentiale eines smarten Regierungs- und Verwaltungshandelns zur Information und Analyse wie zur Automation und Kontrolle sind für viele Bereiche des öffentlichen Sektors bisher noch nicht detailliert erschlossen worden. Dabei eröffnen sich für Ministerien, Ämter und Behörden zahlreiche Möglichkeiten, die im vergangenen Jahrhundert noch zur "Science Fiction“ gezählt wurden: Mitdenkende Ausrüstungen, intelligente Fußfesseln, Überwachungsdrohnen, erweiternde Prüfbrillen für Abnahmen, gemeinsame Aktenbearbeitung oder offene Steuerungscockpits. Auf Grund des rasanten technischen Fortschritts, der Einfachheit in der Bedienung und der Offenheit der Datenbestände erscheint eine substantielle Auseinandersetzung auf einem verständlichen wie nachvollziehbarem Niveau erforderlich. Dabei kann es nicht nur um die Chancen gehen, die bisher noch viel zu kurz kommen. Auch wecken die skizzierten Informations-, Analyse-, Automations- und Kontrollmöglichkeiten in der Bevölkerung und bei Datenschützern Sorgen und Ängste, die ohne eine angemessene Aufbereitung, Diskussion und bewusster Grenzziehung zu Disputen und Konflikten führen. Dass eine detailliertere Analyse und eine anschließende öffentliche Diskussion nicht weiter aufgeschoben werden sollte, lässt sich auch daran verdeutlichen, dass Behörden bereits heute Digitalkameras mit automatischer Zeit- und Geokodierung, smarte Brillen, elektronische Fußfesseln oder frei fliegende Drohnen beschaffen können. Gefragt sind derzeit weder Utopien noch Dystopien, sondern realistische Skizzen, die vor allem dem inhaltlichen Verständnis und dem Diskurs dienen.

Die folgenden sechs Szenarien zu Verwaltung 4.0 sind das Ergebnis mehrerer Workshops an der Zeppelin Universität in Friedrichshafen und bei Fraunhofer FOKUS in Berlin. Diese Szenarien veranschaulichen das breite Gestaltungspotential, das sich aus den Konzepten zu CPS, zum Internet der Dinge und zum Internet der Dienste für ausgewählte Bereiche des öffentlichen Sektors bereits ergibt. Als Skizzen bieten sie einen ersten Überblick, auf dem in Diskussionen, detaillierten Konzeptionen, Prototypentwicklungen und pilothaften Umsetzungen aufgesetzt werden kann. Sie gehen auch das Risiko ein, bewusst anzuecken. Szenarien für bisher noch nicht berücksichtigte Fachverwaltungen werden folgen müssen, denn auch hier besteht Gestaltungsbedarf. Dabei sollte man nicht der falschen Einschätzung erliegen, dass intelligente Objekte und CPS viel zu komplex für die vorhandene schmalbandige Netzinfrastruktur seien. Das taktile Internet (VDE-ITG 2014) als nächste Entwicklungsstufe des Internet wird dafür sorgen, dass in einem Jahrzehnt bereits ein smartes Regierungs- und Verwaltungshandeln über Gigabit-breitbandige Netzwerke in Echtzeit erfolgen kann. 


\subsection{Feuerwehr 4.0 - Lösungsansätze für Freiwillige und Berufsfeuerwehr}

Die Freiwillige Feuerwehr und die Berufsfeuerwehr wehren mögliche Gefahren für Leib, Leben und Sachwerte in Fällen von Bränden und Explosionen ab. Sie helfen auch bei Überschwemmungen, Unfällen und Einstürzen. Zudem kümmern sie sich um den vorbeugenden Brandschutz von Gebäuden. Zum Teil übernehmen sie ebenso Aufgaben der Rettungs- und Krankentransportdienste.

Intelligent vernetzte Objekte wie Rauchmelder, Smartphones, Überwachungskameras und Drohnen können die Arbeit der Feuerwehr unterstützen, etwa wenn Brandherde zu bekämpfen und Nachbargebäude zu überwachen sind. Die integrierten Sensoren erfassen bestimmte Zustände automatisch und melden diese gleich an die Leitzentrale. Smarte Brillen und Digitaldisplays in Feuerwehrhelmen versorgen Einsatzkräfte mit Zusatzinformationen und Vorhersagen, die ihnen Orientierung bieten, etwa bei der Suche nach der Brandmelderzentrale (BMZ) eines Gebäudes, und so wertvolle Unterstützung im Einsatz leisten. Intelligente Kleidung kann in gefährlichen Situationen rasch warnen.

Feuerwehren 4.0 werden verstärkt auf CPS wie etwa Brandschutzanlagen zur Steuerung von Einsatzkräften setzen. In Notfällen können Rettungshelfer und Ordnungskräfte direkt geortet und in Großschadenslagen aus der Ferne gesteuert werden. Intelligent vernetzte Assistenten unterstützen vor Ort. Herausfordernde und unvorhersehbare Einsätze lassen sich so besser vorbereiten. Autonome Roboter und Drohnen sind überall dort zu verwenden, wo es für Menschen zu gefährlich ist.

Rettungskräfte und Feuerwehrleute profitieren von Touchpads und intelligent vernetzten Brillen, weil sie sich bereits auf der Fahrt an den Einsatzort auf ihren Einsatz und dessen Risiken vorbereiten können. So gibt es Apps, die darüber informieren, wo ein befreiender Rettungsschnitt an einem verunglückten Kraftfahrzeug vorgenommen werden muss, um schnellstmöglich Menschenleben zu retten. Apps werten vorhandene Karten, Geoinformationssysteme, Datenbanken und Nachschlagewerke aus, selektieren relevante Informationen und erleichtern so die Planung und Entscheidungsfindung im Einsatz. Die richtige Informationsgrundlage kann Menschenleben retten.

\section{Information und Analyse}

Verhaltensverfolgung

- Erfassung \& Ortung von Rettungskräften: Armband, Uhr, Smartphone \& Wearables

- Überwachungsdrohnen zur Beobachtung \& Messung bei Gefahren \& Brandherden

Verbesserte Situationswahrnehmung

- Intelligent vernetzte Feuerwehrbrille und -helme mit Informationen zum Einsatzort, zur Gefahrenlage \& zur Einsatzsteuerung

- Auswertung der Brandmelderzentrale

Sensorgestützte Entscheidungsanalysen

- Intelligente Kleidung mit Warnfunktion bei gefährlicher Hitze \& Gaskonzentration

- Analyse der Gefährdungslage mit zeitigen Vorschlägen zur Gefahrenbeseitigung

\section{Automation und Steuerung}

Prozessoptimierungen

- Rasche geobasierte Ortung und Steuerung von Einsatzkräften in Notfällen

- Anforderung von Spezialkräften bei Bedarf

- Warnung vor Großschadenereignissen

Optimierter Ressourcenverbrauch

- Einsatzplanungen und Einsatzvorhersagen

- Intelligent eingreifendes Einsatzlagezentrum

- Tablets mit Informationen \& Apps für Einsatz

- Intelligent vernetzter Feuerwehrschlauch

Komplexe autonome Systeme

- Steuerung chaotischer Menschenströme bei Großereignissen und Großschäden

- Autonome Roboter und Drohnen bei gefährlichen Rettungseinsätzen

Tabelle 2: Feuerwehr 4.0: Neuartige Gestaltungsoptionen durch das Internet der Dinge 


\subsection{Gerichte 4.0 und Justizverwaltung 4.0 - Lösungsansätze für die Justiz}

Die Rechtspflegeorgane sind für die Rechtsprechung zuständig. Richter verhandeln in Gerichten die Fälle mit Staatsanwaltschaften und Rechtsanwälten. Die Justizverwaltung schafft die sachlichen und personellen Voraussetzungen für eine geordnete Rechtspflege. Zugleich führt sie die Dienstaufsicht über die Organe der Rechtspflege, das Strafregister, die Handelsregister, die Grundbücher und den Strafvollzug mit Gefängnissen und Bewährungshilfen.

Intelligente Objekte und CPS werden überall dort Verwendung finden, wo sie Richtern, Staatsanwälten und Justizbeamten eine effizientere Wahrnehmung ihrer Aufgaben versprechen. Ein intelligent vernetztes Register oder Grundbuch informiert Berechtigte auf Wunsch proaktiv über Veränderungen, soweit solche Meldungen rechtlich zulässig sind. Elektronisch überwachbare Fußfesseln eignen sich bei harmlosen Straftätern für neuartige Formen des räumlich begrenzten Hausarrests. Verlässt ein Delinquent eine bestimmte Zone, wird ihm dies warnend signalisiert. Reagiert er nicht, wird die Verwaltung rasch aktiv. Dies entlastet den Strafvollzug personell und finanziell. Das Bewegungsverhalten von Personen im offenen Vollzug ließe sich alternativ mit intelligenten Armbändern, Uhren und Smartphones verfolgen. Richter profitieren von smarten Richterarbeitsplätzen und vernetzten Fallakten, die bei neuen Sachständen automatisch aktualisiert und visuell verständlich aufbereitet werden. Intelligent vernetzte Cockpits erleichtern dem Gerichtspersonal, Gefängnispersonal und Bewährungshelfern durch aktuelle Informationsaufbereitung ihre Arbeit. Funktionieren kollaborative Fallakten und der elektronische Rechtsverkehr, ist eine ganz neue Form der Rechtsprechung vorstellbar. Richter, Staatsanwaltschaft und Rechtsanwälte hätten gleichzeitig Zugriff auf die Akte und könnten über sie gemeinsam an einem Vergleich oder an der Rechtsprechung arbeiten. Werden die Dokumente des Urteils und des Strafbefehls mit Zusatzinformationen erweitert, lassen sich Rechtsprechungsdatenbanken zeitnah aktualisieren und Prozesse des Strafvollzugs optimieren. Die konsequente Vernetzung erlaubt zudem einen grenzüberschreitenden Verbund elektronischer Handelsregister und Grundbücher in Wirtschaftsräumen und dem digitalen europäischen Binnenmarkt.

\section{Information und Analyse}

Verhaltensverfolgung

- Erfassung: Armband, Uhr \& Smartphone

- Intelligente vernetzte Fußfessel

- Intelligent vernetztes Register

- Intelligent vernetztes Grundbuch

Verbesserte Situationswahrnehmung

- Intelligent vernetzter Richterarbeitsplatz

- Gerichtscockpit für Gerichtsverwaltung

- Rasch informierte Bewährungshilfe bei Überwachung der Auflagenerfüllung

Sensorgestützte Entscheidungsanalysen

- Lügendetektoren

- Justizvollzugscockpit im Gefängnis

- Auf Situationen intelligent reagierende Hand- und Fußfesseln

\section{Automation und Steuerung}

Prozessoptimierungen

- Gemeinsame Fallakte im Gerichtsverfahren

- Smartes Urteilsdokument (mit Beschlüssen)

- Smarte Rechtsprechungsdatenbank

- Intelligent vernetzter Strafbefehl

Optimierter Ressourcenverbrauch

- Planungen zur Inhaftierung Verurteilter mit Vorhersagen zu kritischen Situationen

- $\quad$ Automatische Überwachung der Einhaltung von bestimmten Bewährungsauflagen

Komplexe autonome Systeme

- Elektronischer Rechtsverkehr

- Verbund elektronischer Handelsregister

- Intelligent vernetztes Gefängnis (Smart Jail)

- Intelligent vernetzte Bewährungshilfe

Tabelle 3: Justizverwaltung 4.0: Neuartige Gestaltungsoptionen durch das Internet der Dinge 


\subsection{Finanzverwaltung 4.0 - Lösungsansätze für das Steuerwesen}

Gebietskörperschaften aller Ebenen erheben im verfassungsrechtlichen Rahmen eigene Steuern zur Erledigung ihrer öffentlichen Aufgaben. Im Kontext von Steuererhebung, Steuererklärung, Steuerberechnung und Steuerrückerstattung eröffnen sich für intelligent vernetzte Objekte und CPS neuartige Ansätze für eine sehr effiziente Finanzverwaltung 4.0.

Zur Sicherung des Steueraufkommens werden Steuerbeträge aus der Steuerquelle automatisch abgeführt, etwa im Quellenabzugsverfahren direkt bei Arbeitgebern oder Banken. Werden Steuern automatisiert zum Zeitpunkt einer Transaktion erhoben, sollten die Steuerüberweisung und die dazugehörige informierende Steuermeldung an die Steuerverwaltung direkt generiert werden können. Vertraut die Steuerbehörde dem Steuerzahler, ist eine Bündelung der abzuführenden Steuern über eine Buchungstransaktion am Tag, in der Woche, im Monat oder im Quartal vorstellbar. Eingehende Zahlungen sollten innerhalb der Steuerbehörde automatisch verbucht und dem jeweiligen Steuerkonto zugerechnet werden können. Elektronische Steuererklärungen ersetzten die papierbasierten Formulare, denn sie rufen die vorhandenen erhebungsrelevanten Daten automatisiert ab, prüfen eingegebene Daten auf Unstimmigkeiten und weisen auf Optimierungen hin. Intelligent vernetzte Steuerakten unterstützen Bürger, Steuerberater und Finanzbeamten bei der Bearbeitung von Steuerkonto, Steuererklärung, Steuerbelegen (intelligente Rechnungen), Steuerberechnung und automatischem Steuereinzug. Ein smarter Steuerbescheid sucht sich selbst den Weg zum Steuerpflichtigen, erlaubt eine automatische Prüfung seiner Inhalte und kann auf Wunsch oder Anforderung in weiteren Antrags- und Prüfverfahren direkt verwendet werden. Die Leitungsebene wird sich über analysierende Cockpits einen aktuellen Überblick zu Bearbeitungsständen und Steueraufkommen verschaffen wollen. Eine automatisierte Auswahl prüfungswürdiger Fälle kann die Prüfungsvorgänge optimieren, das Risikomanagement der Steuerbehörden verbessern und die Betrugsbekämpfung stärken. Im Kampf gegen Steuerhinterziehung werden Staaten künftig weltweit enger zusammenarbeiten, sich austauschen, global vernetzen und gemeinsam gegen Steuerbetrug vorgehen.

\section{Information und Analyse}

Verhaltensverfolgung

- Meldungen an die Steuerverwaltung

- Einreichungen von Steuererklärungen

- Überweisungen an die Steuerkassen

- Veröffentlichungen von Bilanzen

Verbesserte Situationswahrnehmung

- Intelligent vernetzte Steuerakte

- Intelligent vernetztes Steuerkonto

- Portal zur Steuerprüfung mit Vorprüfung

- Interne Cockpits für die Leitungsebene

\section{Sensorgestützte Entscheidungsanalysen}

- Wirksames Risikomanagement und vorbeugende Betrugsbekämpfung durch gezielte und automatisierte Auswahl prüfungswürdiger Fälle

\section{Automation und Steuerung}

Prozessoptimierungen

- Elektronische Steuererklärung mit Vorausfüllung vorliegender Steuerdaten

- Smarter Steuerbescheid (Objekt)

- Automatisierte Verbuchung von Zahlungen

Optimierter Ressourcenverbrauch

- Automatisierte Steuermeldung zum Zeitpunkt des Besteuerungstatbestandes

- Einfache Steuerprüfung als Normalfall

- Gezielte Steuerprüfung bei Verdacht

Komplexe autonome Systeme

- Autonomes Steuererhebungssystem unter Einbindung von Steuerakte und Steuerkonto

- Steuerprüfung aus der Ferne

- Globales Steuerbetrugserkennungssystem

Tabelle 4: Finanzverwaltung 4.0: Neuartige Gestaltungsoptionen durch das Internet der Dinge 


\subsection{Standesamt 4.0 - Lösungsansätze für dlas Personenstandswesen}

Das Personenstandswesen wird von den Standesämtern geführt. Die bewährten Personenstandsbücher sind mittlerweile in elektronische Personenstandsregister überführt worden. Nun werden Geburten, Eheschließungen, Lebenspartnerschaften und Todesfälle digital festgehalten. Die Register übernehmen damit die Funktionen von intelligent vernetzten Personenstandsbüchern. Dies erlaubt es jederzeit, Übersichten über eingegangene Meldungen eines oder mehrerer Standesämter zu erstellen und bei Bedarf zu analysieren. Eine intelligente Vernetzung von Registern und weiteren Informationssystemen gestattet es Standesbeamten ihre persönlichen Auskunfts- und Beratungsgespräche zur Anmeldung der Eheschließung umfassender durchzuführen. Ergänzend zum Gesamtportfolio könnten sie auch vorhandene Räumlichkeiten vorstellen, Termine verbindlich zusagen und Eheverbotsprüfungen bald in Echtzeit über elektronische Registerabfragen vornehmen. Technisch vorstellbar, aber ethisch nicht wünschenswert, wären DNA-Partnerabgleiche mit Hilfe genetischer Datenbanken, um zu überprüfen, ob andere gewichtige Gründe gegen eine Eheschließung sprechen.

Nachhaltige Prozessoptimierungen versprechen smarte elektronische Geburts-, Eheschließungs-, Lebenspartnerschafts- und Sterbeurkunden. Als eigenständig ansprechbare Objekte geben sie Auskunft über den Stand einer Person. Auf Wunsch könnten sie weitere Prozesse anstoßen, so dass ganz im Sinne einheitlicher Ansprechpartner mit einer Meldung oder Anzeige im Standesamt auch alle weiteren Amtsgänge initiiert und erledigt werden könnten. Solche Rundum-Pakete, die in Kooperation mit Krankenhäusern, Bestattungsunternehmen und anderen vertrauensvollen Einrichtungen angeboten werden könnten, entlasten Eltern, Ehepartner und Hinterbliebene. Intelligent vernetzte Ehefähigkeitszeugnisse entlasten Standesbeamte, da sich automatisiert die Richtigkeit der Inhalte und das Vertrauen in die ausstellende Stelle überprüfen lassen. Vertrauen sich Standesbeamte, Standesämter und Staaten gegenseitig, sollten sie sich national oder global miteinander elektronisch vernetzen. Sobald Register und Standesämter direkt miteinander elektronisch kommunizieren, kann etwa auf papierbasierte Urkunden und amtlich beglaubigte Übersetzungen verzichtet werden.

\section{Information und Analyse}

Verhaltensverfolgung

- Aktuelle Übersichten und Analyse: Geburten, Eheschließungen, Lebenspartnerschaften und Sterbefälle

Verbesserte Situationswahrnehmung

- Umfassendes persönliches Auskunfts- \& Beratungsgespräch im Standesamt

- Visualisierung des Gesamtportfolios

- Intelligente Ermittlung von Eheverboten

Sensorgestützte Entscheidungsanalysen

- Spontane Organisation von Feierlichkeiten um die Eheschließung in Verbindung mit den Wetterberichten

- Abgleiche über genetische Datenbanken

\section{Automation und Steuerung}

Prozessoptimierungen

- Smarte Urkunden des Standesamts (Objekt): Von Geburtsurkunde bis zur Sterbeurkunde

- Meldung einer Geburt (Rundum-Paket)

- Anzeige eines Sterbefalles (Rundum-Paket)

Optimierter Ressourcenverbrauch

- Vereinfachte Überprüfung dank intelligent vernetzter Ehefähigkeitszeugnisse

- Strukturierte elektronische Kommunikation zwischen Standesämtern

Komplexe autonome Systeme

- Intelligent vernetztes Standesamt

- Verbund der Personenstandsregister

- Globaler Verbund der Standesämter

- Offenes Hochzeitsportal mit Schnittstellen

Tabelle 5: Standesamt 4.0: Neuartige Gestaltungsoptionen durch das Internet der Dinge 


\subsection{Landwirtschaft 4.0 - Lösungsansätze fürr Landwirtschaftsverwaltung}

Für die Agrar- und Viehzuchtindustrie eröffnet die Landwirtschaft 4.0 neuartige Möglichkeiten. Smarte Bauernhöfe lassen sich mit intelligent vernetzten Objekten und CPS effizienter managen, Felder, Weinstöcke und Wälder präziser bewirtschaften und Viehstallungen besser kontrollieren. Intelligente Assistenzsysteme mit Anbindung an meteorologische Dienste unterstützen die Bauern bei den richtigen Saat-, Dünge- und Ernteentscheidungen. In Deutschland ist die staatliche Landwirtschafts-und Weinbauverwaltung jedoch kaum noch als Produzent aktiv. Vielmehr kümmert sie sich um die Belange der Landwirte, Züchter, Forstwirte, Winzer und Fischer, der verarbeitenden Betriebe der Lebensmittelindustrie wie der Verbraucher und greift regulierend ein.

RFID-Chips in Nutztieren, Kisten und Containern erlauben es, Herkunft und Verarbeitung von landwirtschaftlichen Produkten über die gesamte Lieferkette hinweg zu verfolgen. Über zunehmend ausgereiftere cyberphysische Lieferkettensysteme lassen sich Herkunft, Futtermittel, Schlachtung, Verarbeitung, Vertrieb und Zubereitung in Gaststätten verfolgen. Gesetzlich vorgeschriebene Datenerhebungen, Meldungen und Kontrollen könnten mit Hilfe intelligenter Sensoren automatisch vorgenommen und transparent veröffentlicht werden. So lassen sich Vorreiter auszeichnen, Missstände, Krankheiten und Seuchen rascher entdecken und mit amtsärztlichem Personal zügiger bekämpfen und beseitigen. Mit Siegeln und Zertifikaten werden vorbildhafte transparente Lieferketten und -netzwerke prämiert. So kann ein nachhaltiges Vertrauen bei den Verbrauchern aufgebaut werden. Portale helfen Förderprogramme besser zu erschließen und Subventionszahlungen nachzuvollziehen.

Allerdings kann in komplexen Wertschöpfungsketten aus der Qualität der Arbeit eines konkreten Anbieters immer weniger auf die Produktionsbedingungen und Qualität des Gesamtproduktes geschlossen werden. Im Sinne des Verbraucherschutzes ist die gesamte Wertschöpfungskette mit allen Materialflüssen zu erfassen und zu visualisieren. Intelligente Lieferketten stellen so zu Produkten und Dienstleistungen die Einhaltung sozialer, ökologischer und hygienischer Standards sicher.

\section{Information und Analyse}

Verhaltensverfolgung

- Herkunfts- und Erzeugungstracking

- Produktionstracking

- Liefer- und Handelstracking

- Subventionstracking

Verbesserte Situationswahrnehmung

- Drohnen zur Überwachung der Felder

- Cockpit für die Bewirtschaftung der Felder

- Cockpit für die Bewirtschaftung der Ställe

- Monitoring von Krankheiten und Seuchen

Sensorgestützte Entscheidungsanalysen

- Übersichten und Analysen zum Viehbestand durch Signale der RFID-Chips

- Übersichten und Vorschläge zum Einsatz von Düngermitteln

\section{Automation und Steuerung}

Prozessoptimierungen

- Intelligent vernetzter Herkunftsnachweise zu landwirtschaftlichen Erzeugnissen

- Intelligent vernetzte Lebensmittelkontrollen

- Smartes Ernten mit smarten Traktoren

Optimierter Ressourcenverbrauch

- Smarte Bewirtschaftung der Felder

- Smarte Bewirtschaftung der Ställe

- Intelligent vernetzte Nahrungsmittelversorgung in Zeiten knapper Ressourcen
Komplexe autonome Systeme

- Intelligent vernetzte Bauernhöfe

- Intelligent vernetzte Ställe

- Intelligente Liefer- und Wertschöpfungskette

- Fernveterinärversorgung

Tabelle 6: Landwirtschaft 4.0: Neuartige Gestaltungsoptionen durch das Internet der Dinge 


\subsection{Bauverwaltung 4.0 - Lösungsansätze für Hochbau und Tiefbau}

Straßen, Autobahnen, Eisenbahnen, Flüsse, Kanäle und Brücken sind zentrale Pfeiler die Verkehrsinfrastruktur eines Staates. Durch die tägliche Inanspruchnahme nutzen sie sich mit der Zeit ab. Daher müssen sie in regelmäßigen Abständen überprüft, gegebenenfalls überholt oder ersetzt werden. Sensoren vervollkommnen Verkehrsinfrastrukturen, indem diese automatisch über Inanspruchnahme und Zustand berichten. Mit Sensoren und Aktoren lassen sich auch Gebäude zum smarten Haus ausbauen, die autonom Jalousien, Licht, Heizung und Hausgeräte steuern.

Tiefbauverwaltungen beseitigen erkannte Schäden an der Verkehrsinfrastruktur, um einen optimalen Verkehrsfluss zu ermöglichen. Sensorgenerierte Informationen zur Belastung von Straßen und Brücken helfen ihnen, den Infrastrukturzustand besser einzuschätzen. Direkte Angaben der Bürger und von Smartphones registrierte Starkvibrationen bei Autofahrten vereinfachen die frühzeitige Identifikation und Beseitigung von Straßenschäden. Hochbauverwaltungen kümmern sich dagegen um den Zustand öffentlicher Gebäude, die sich durch Sensoren- und Aktorennetzwerke funktional erweitern und besser managen lassen. Im intelligent vernetzten Gebäude können dank CPS diverse manuelle Aufgaben automatisiert und Ressourcenverbrauche optimiert werden. Der elektronische Baurechtsverkehr eröffnet Chancen für Abläufe in technischen Rathäusern, um Bauanträge gemeinsam transparenter und effizienter zu bearbeiten. Großes Potential steckt in intelligenten Prüfbrillen für Bauabnahmen, wenn Prüfer Plan, Realität und einzuhaltende technische Standards in einem Blick haben.

Tunnel gelten als neuralgische Punkte der Verkehrsinfrastruktur. Störungen durch Fehlfunktionen oder äußere Einwirkungen bedrohen nachhaltig die Funktionsfähigkeit der gesamten Infrastruktur. Zugleich besteht eine starke Gefährdung für die Personen im Tunnel. Rettungsmaßnahmen müssen daher schnell und umfassend mit geeigneten Instrumenten durchgeführt werden. Vielfältige, robuste und redundant arbeitende Sensoren- und Aktorennetzwerke bieten die Möglichkeit, die Situationswahrnehmung deutlich zu verbessern und vor Ort die richtigen, rettenden Entscheidungen zu treffen.

\section{Information und Analyse}

Verhaltensverfolgung

- Belastung von Straßen und Brücken

- Identifizierung von Straßenschäden durch Vibrationshinweise von Smartphones

- Fortschritte bei Bauvorhaben

\section{Verbesserte Situationswahrnehmung}

- Intelligente Prüfbrille für Bauabnahmen, die Pläne und einzuhaltende Standards ins Blickfeld des Gutachters bringen

- Drohnen zur Abnahme von Hochbauten

Sensorgestützte Entscheidungsanalysen

- Sensorgestützte Belastungsprüfungen von Verkehrsinfrastrukturen

- Unterstützung baustatischer Prüfungen bei Abnahme von Hoch- und Tiefbauten

\section{Automation und Steuerung}

Prozessoptimierungen

- Automatisierte Ableseaufgaben

- Halbautomatisierte Gebäudesteuerung

- Zeitnahe Schadenserfassung \& -beseitigung

- Gemeinsame Bearbeitung von Bauanträgen

Optimierter Ressourcenverbrauch

- Minimierung des Verbrauchs von Strom, Heizöl und Erdgas für ein Gebäude

- Ablaufoptimierte Einbindung zuständiger Stellen im Rahmen eines Bauantrags

Komplexe autonome Systeme

- Elektronischer Baurechtsverkehr

- Intelligent vernetzte Gebäude

- Intelligent vernetzte Straßen

- Intelligent vernetzte Tunnel 


\section{Schritte auf dem Weg zur intelligent vernetzten Verwaltung 4.0}

\subsection{Forschungsagenda für "Smart Government" und "Verwaltung 4.0"}

Ausgehend von dem Verständnis eines intelligent vernetzten Regierungs- und Verwaltungshandelns („Smart Government“) und dem dazugehörigen Leitbild „Verwaltung 4.0“ stellt sich zum derzeitigen Zeitpunkt die Frage nach der weiteren inhaltlichen Konkretisierung über eine Forschungsagenda. Gefragt sind Vorschläge der Wissenschaft, um mit Unterstützung und in Partnerschaft mit öffentlicher Verwaltung, der Wirtschaft und Bürgern sich den zentralen Fragestellungen von Smart Government zu nähern und um die vielfältigen offenen Fragen einer Realisierung bestmöglich zu beantworten.

Im Sinne einer gestaltungorientierten Forschung geht es um die inhaltliche Gestaltung intelligent vernetzter Objekte, CPS und darauf aufsetzender Anwendungsszenarien im öffentlichen Sektor. Zunächst gilt es zu klären, welche Ansätze smarter Objekte sich für den Einsatz im öffentlichen Sektor eignen. Mit Funkchips, Sensoren und Aktoren ausgestattete Objekte, die untereinander kommunizieren, Zustände wahrnehmen und begrenzt reagieren, eröffnen der öffentlichen Verwaltung teils neuartige Möglichkeiten zur Aufgabenerledigung, die herkömmlichen Ansätzen in Geschwindigkeit, Qualität, Kosten und Ergebnis überlegen sind. Zu entwickeln sind etwa vertrauensvolle smarte Akten, Bescheide, Urkunden und Register, aber auch smarte Prüfbrillen und Überwachungsdrohnen, wenn diese der öffentlichen Aufgabenerfüllung dienen. Durch die Sensoren und deren Vernetzung fallen große Mengen an Sensordaten an. Eine intelligente Auswertung dieser Daten eröffnet Raum für hochwertige Analysen und Prognosen wie zur Automation und Steuerung. Herausforderung ist es, mit Blick auf die Sorge vor einem Überwachungsstaat diese smarten Objekte datenschutzkonform und vor unberechtigten Zugriffen sicher geschützt zu konstruieren.

Zweitens stellt sich die Frage, welche und vor allem wie vertrauenswürdige und verlässliche CPS für den öffentlichen Sektor zu konzipieren, zu bauen, zu vernetzen, zu steuern, zu kontrollieren und zu warten sind (vgl. acatech 2011, S. 5). Über Sensornetze, Aktorennetze und Webdienste kann im großen Umfang und über räumliche Entfernungen hinweg auf zahlreiche smarte Objekte zugegriffen werden. Dadurch wird es möglich, Frühwarnsysteme mit überschaubarem Aufwand zu realisieren. Mit Blick auf die Breite und die Aufgabenvielfalt des öffentlichen Sektors sowie vorhandene Probleme und Herausforderungen gilt es vor allem neuartige CPS zu entwickeln, die durch bessere Information, hochwertige Datenanalyse, effiziente Automation und selbststeuernde Kontrolle erheblich zur effizienteren öffentlichen Aufgabenerledigung beitragen. Die ersten Skizzen zu Einsatzlagezentren, zur Bewährungshilfe, zur Steuerbetrugserkennung, zu Standesämtern, zu Bauprüfämtern und anderen Aufgabenfeldern für CPS gilt es inhaltlich zu vertiefen. Eine angemessene Einbindung in das Internet der Systeme, der Menschen und der Daten hat dabei unbedingt zu erfolgen.

Drittens ist zu prüfen, welche bereits vorhandenen smarten Objekte wie Smart Watch, Smart Phone, Smart Pad oder Smart TV sich zur Aufgabenerfüllung in Staat und Verwaltung eignen. Zugleich sollte reflektiert werden, bis zu welchen Grenzen eine Verwendung akzeptabel und wo aus berechtigten Gründen davon Abstand zu nehmen wäre. Dieselbe Fragestellung muss auch mit Blick auf den Einsatz vorhandener CPS wie Frühwarnsysteme zur Verwendung in Staat und Verwaltung gestellt werden.

Die vorgestellten sechs Szenarien zu Smart Government in Kapitel 4 zeigen, wie sich das Leitbild „Verwaltung 4.0" unterschiedlich konkretisieren lässt. Mit Blick auf die Aufgabenvielfalt des öffentlichen 
Sektors sind weitere Szenarien für andere Fachbereiche zu entwickeln, übrigens auch für den ländlichen Raum und Entwicklungsländer, um ein Gespür für das Potential des Internet der Dinge und der Dienste für und im öffentlichen Sektor zu gewinnen. Dennoch muss auch überlegt werden, ob und in welchem Umfang vom Einsatz intelligenter Vernetzung in bestimmten Bereichen prinzipiell Abstand zu nehmen ist, etwa in denen Waffen zum Einsatz und dabei auch Menschen zu Schaden kommen.

Im Internet der Dienste werden Dienste und Funktionalitäten als feingranulare Softwarekomponenten abgebildet und von den Bereitstellern auf Anforderung zur Verfügung gestellt. Die einzelnen für den öffentlichen Sektor noch zu konkretisierenden Softwarebausteine sind als webbasierte Dienstleistungen miteinander integrierbar. Organisationen können modular einzelne Softwarekomponenten zu komplexen und dennoch flexiblen Lösungen orchestrieren. Einzelne Verwaltungsprozesse lassen sich in einem innovativen Gesamtangebot bündeln. Portale ermöglichen sowohl Verwaltungsleistungen aus einer Hand als auch neuartige privatwirtschaftliche Dienstangebote mit integrierten Verwaltungsprozessen. Bürger und Unternehmen werden solche portalbasierte einheitlichen Ansprechpartner schätzen, die sich um alles kümmern und sie so vor eigenen Amtsgängen, der Suche nach der zuständigen Stelle und bürokratischen Aufgaben bewahren. Aus ökonomischen Erwägungen heraus macht es Sinn, dass nicht jede Gebietskörperschaft sämtliche Dienste separat entwickelt und vorhält. Insofern muss über interoperable Architekturen und die Konzeption neuartiger Dienstleistungszentren nachgedacht werden, die über Kreis- und Landesgrenzen hinweg wirken.

Aus einer juristischen Perspektive gilt es zu prüfen, ob es für Smart Government im Allgemeinen und den konkreten Einsatz eines intelligent vernetzten Objekts im öffentlichen Sektor im Besonderen eine bereits ausreichende Rechtsgrundlage gibt und was diese zulässt. Auch aus den CPS lassen sich ITrechtliche und datenschutzrechtliche Fragestellungen ableiten. Zudem ergibt sich aus dem Internet der Dinge und dem Internet der Dienste weiterer Regelungsbedarf. Mit Blick auf zahlreiche ungeklärte rechtliche Fragestellungen gilt es den Rechtsgestaltungsbedarf durch den Gesetzgeber zu bestimmen und mit in der Sache förderlichen Vorschlägen zu konkretisieren. Erste juristische Symposien widmen sich bereits den Herausforderungen der weiterreichenden intelligenten Vernetzung.

Für die empirische Sozialforschung stellt sich zu diesem frühen Zeitpunkt die Frage, ob es Personen und Personengruppen gibt, die sich mit all diesen Fragestellungen schon angemessen auseinander setzen, und wie diese die weitere Entwicklung sehen und beeinflussen. Mit Literaturstudium, Beobachtungen und Experteninterviews werden sie zu Erkenntnissen kommen, in welche Richtungen sich eine intelligent vernetztes Regierungs- und Verwaltungshandeln entwickeln kann, welche Stärken und Schwächen damit verbunden, welche Chancen und Risiken zu berücksichtigen sowie welche Handlungsempfehlungen aus Sicht der Experten zu geben sind. Zugleich geht es um Akzeptanz der Technologien und Veränderungen der Prozesse und Strukturen. Besonders wünschenswert wären Vorschläge zu einem bürgerorientierten Smart Government, das Bürger aktiv mitgestalten dürfen. 


\subsection{Aktuelle Herausforderungen}

In Deutschland sind ein "intelligent vernetztes Regierungs- und Verwaltungshandeln“ („Smart Government") und "Verwaltung 4.0" noch keine etablierten Schlagwörter. Konkretisierungen der Begriffe „smarte Behörde“, „smarte Bescheide“, „smarte Beamte“ und „smarte Bürger" stehen noch aus. Noch fehlen Leitbilder zum Umgang von Staat und Verwaltung mit dem Internet der Dinge und dem Internet der Dienste. Bund, Länder und Kommunen müssen sich in einer ebenenübergreifenden Arbeitsgruppe diesen Fragen stellen und ihre eigenen Definitionen erarbeiten. Dies passiert bisher noch nicht. Der Schritt zur Verwaltung 4.0 und einer umfassenden intelligenten Vernetzung kann eigentlich nur im gemeinsamen Dialog von Politik und Verwaltung mit Wissenschaft, Wirtschaft und Zivilgesellschaft gelingen. Schließlich werden alle Gruppen von der intelligenten Vernetzung in Staat und Verwaltung betroffen sein und ihre Vorstellungen einbringen wollen.

Dieser Weg sollte rasch eingeschlagen werden, denn die technische Entwicklung schreitet voran. Die Wissenschaft sollte sich darum bemühen, die Vision weiter zu konkretisieren und eine Forschungsagenda (Vorschlag: Abschnitt 5.1) zu formulieren. Mit Blick auf Vielfalt und Komplexität muss eine multi- und interdisziplinäre Auseinandersetzung mit smarten Ansätzen in Staat und Verwaltung eingefordert werden. Politik und Verwaltung werden eigene Vorstellungen in Konzeption, Datenschutz, Gesetzgebung und Umsetzung einbringen. Die Wirtschaft sollte ihre technische und ökonomische Expertise beisteuern, etwa bei der Standardisierung, dem Breitbandausbau, der Sicherheitsinfrastruktur und spezifischen Produktinnovationen. Auch eine Einbindung zivilgesellschaftlicher Akteure erscheint unerlässlich, denn konstruktive Vorschläge, Bedenken und Kritik aus der Bevölkerung sind im Zeitalter zunehmender Bürgerbeteiligung zum Aufbau von Vertrauen und Akzeptanz insbesondere bei so bedeutenden Infrastrukturkomponenten angemessen zu berücksichtigen

Gerade das disruptive Potential von intelligent vernetzten Objekten und CPS machen eine intensivere inhaltliche Auseinandersetzung und einen gesellschaftlichen Diskurs über Smart Government erforderlich. So gilt es zu bestimmen, in wieweit eine IT-basierte Verhaltensverfolgung, eine verbesserte Situationswahrnehmung und sensorgestützte Entscheidungsanalysen akzeptabel sind, ohne dass dies als Überwachungs- und Unterdrückungsmaßnahmen wahrgenommen werden. Ebenso muss reflektiert werden, ob weiterhin alle Entscheidungen von Menschen zu treffen sind oder ob in bestimmten Bereichen autonome IT-Systeme eigenständig und automatisch Entscheidungen treffen und diese umsetzen dürfen. Schon aus diesen Fragestellungen ergibt sich ein Aus- und Weiterbildungsbedarf.

Mit Blick auf das hohe Datenschutzniveau in Deutschland wird sich die Bevölkerung sehr kritisch mit intelligent vernetzten Objekten und CPS auseinandersetzen, die regelmäßig Sensordaten erfassen und an Analyse- und Steuerungssysteme weiterleiten. Im Sinne von Datenvermeidung und Datensparsamkeit muss nicht unbedingt auch alles ausprobiert, nicht alles gespeichert und nicht alles ausgewertet werden. Bürger werden Einspruch gegen solche Systeme einlegen, wenn sie sich in ihrem Recht auf informationelle Selbstbestimmung eingeschränkt fühlen und die Gewährleistung von Vertraulichkeit und Integrität ihrer eigenen informationstechnischen Systeme gefährdet sehen. Staat und Verwaltung haben zu akzeptieren, dass nicht alles was technisch möglich wäre, gesellschaftlich akzeptiert und toleriert wird. Vielmehr werden die Bürger ihre Datenhoheit selbst ausüben wollen und über Datenschutzbeauftragte und den Gesetzgeber dem Staat klare Schranken setzen. Dies ist zugleich eine echte Chance für den Standort, von Anfang an von intelligent vernetzten Objekten und CPS die Einhaltung eines vergleichsweise hohen Datenschutzniveaus zu verlangen. 
Unter diesen Rahmenbedingungen wird es für Politik, Verwaltung, Wirtschaft und Wissenschaft eine echte Herausforderung, vertrauenswürdige und verlässliche CPS für den öffentlichen Sektor zu konzipieren, zu bauen, zu vernetzen, zu steuern, zu kontrollieren und zu warten. Smart Government und die Umsetzung des Leitbilds „Verwaltung 4.0" sind sehr anspruchsvolle Vorhaben. Aufbauend auf Definition, Vision und ersten Skizzen müssen Unterstützer und Partner gefunden, Ziele vereinbart, Arbeitspakete für einen Arbeitsplan geschnürt, Ressourcen bereitgestellt und Prototypen entwickelt werden. Personelle und finanzielle Mittel sollten dort zur Erledigung der vereinbarten Aufgaben angemessen bereitgestellt werden, wo diese zur Unterstützung und Realisierung erforderlich sind. Eine inhaltliche Auseinandersetzung „zum Nulltarif“ wird nicht zielführend sein, da zu viele Ergebnisse dann eher vom Zufall, vom Engagement Einzelner und von der verborgenen Agenda der wenigen Sponsoren abhängen, ohne die Bevölkerung ernsthaft wie angemessen mitzunehmen.

\subsection{Warum Deutschland auf Smart Government nicht mehr länger warten sollte}

Ansätze eines smarten Regierungs- und Verwaltungshandelns auf Grundlage einer intelligenten Vernetzung vorhandener und neuer Objekte eröffnen Staat und Verwaltung ganz neue Möglichkeiten. In ihrer Summe können diese zur weiteren Steigerung der Lebensqualität, zur Verbesserung der Standortqualität und zur Stärkung der Bürgerorientierung beitragen. Zugleich eröffnen sie neuartige Chancen zur Informationen und Analyse sowie zur Automation und Kontrolle, die teils positiv und teils negativ bewertet werden. Unabhängig von einer Umsetzung in Staat und Verwaltung werden smarte Technologien von Wissenschaft und Wirtschaft weltweit laufend weiter entwickelt, perfektioniert und marktfähig gemacht. Smarte Objekte und CPS werden dort Verwendung finden, wo sie zu Innovationen führen, Mehrwerte schaffen, Effizienzsteigerungen bieten oder Kosten senken. Steigende Bandbreiten stärken vor allem das Internet als zentrale Kommunikationsplattform.

Allerdings können intelligent vernetzte Lösungsansätze zu disruptiven Veränderungen in Staat und Verwaltung führen, insbesondere wenn die neuen Lösungen und Wertschöpfungsnetzwerke der bisherigen Aufgabenwahrnehmung überlegen sein sollten. Bisher sind viele dieser Möglichkeiten in ihrer Breite und Tiefe sowie die damit verbundenen Veränderungen vielen Meinungsmultiplikatoren in Politik, Verwaltung, Wirtschaft, Wissenschaft und Publizistik weder bekannt noch bewusst. Dies wird sich ändern müssen, denn nur im gemeinsamen konstruktiven Dialog von Politik, Verwaltung, Wissenschaft, Wirtschaft und Zivilgesellschaft kann die inhaltliche Gestaltung von Smart Government erfolgreich angegangen und bewältigt werden.

Vor diesen Herausforderungen stehen derzeit alle Staaten. Weltweit mag es Staaten geben, die sich mit einem anderen Demokratie- und Rechtsstaatverständnis dieser vierten Entwicklungsstufe intelligent vernetzter Objekte nähern und eigene, totalitäre Lösungsansätze entwickeln. Und es wird immer Unternehmen geben, die solche Systeme dann auch planen, implementieren und im staatlichen Auftrag betreiben werden. Mag ein Wettbewerb der Ideen und Umsetzungen noch so bereichernd wirken, smarte Technologien bleiben nur ein Mittel zum Zweck. Sie können zum Guten wie zum Schlechten verwendet werden. Überzeugende Lösungen werden sich jedoch am Markt durchsetzen und so nachhaltig die Pfade zur weiteren Entwicklung vorgeben.

Insofern ist eine inhaltliche Auseinandersetzung mit dem Internet der Dinge und dem Internet der Dienste für öffentliche Aufgaben, mit intelligent vernetzten Objekten und CPS im öffentlichen Sektor dringend geboten. Ansonsten besteht die Gefahr, dass man ohne Kenntnisse und ohne Engagement 
keinerlei Gewicht besitzt und kaum Einfluss auf die Gestaltung entsprechender Informations- und Entscheidungssysteme nehmen kann. Stattdessen müssten sich Staat und Verwaltung dann mit anderswo gestalteten Lösungen begnügen, die ihren Anforderungen nur bedingt entsprechen und oft mit unbeabsichtigten Nebenwirkungen verbunden sind. Daher empfiehlt sich eine schrittweise erfolgende, fundierte inhaltliche Auseinandersetzung mit den Potentialen ausgewählter intelligenter Objekte und CPS, mit ihren Chancen, Risiken, Grenzen und Herausforderungen sowie ein darauf aufsetzendes Arbeitsprogramm zur nutzbringenden wie eigenständigen Erschließung eines smarten Regierungs- und Verwaltungshandelns. Die öffentliche Verwaltung in Deutschland sollte dieses sich derzeit öffnende Zeitfenster zur digitalen Neugestaltung vieler Fachaufgaben und Abläufe und zum Aufbau einer intelligent vernetzten digitalen Verwaltungsinfrastruktur für die anstehenden Jahrzehnte dringend nutzen. Versiegen durch die vorhersehbare demographische Entwicklung erst einmal die finanziellen Ressourcen, wäre es für eine aktive Gestaltung viel zu spät. 


\section{Literatur}

8ITG 2014: Arbeitsgruppe 2 des Nationalen IT-Gipfels „Digitale Infrastrukturen als Enabler für innovative Anwendungen": Digitale Infrastrukturen - Jahrbuch 2013/2014 -

Schwerpunkte und Zielbilder für die Digitale Agenda Deutschlands, 2. Auflage,

Arbeitsgruppe 2 des Nationalen IT-Gipfels, Berlin 2014.

acatech 2011: acatech - Deutsche Akademie der Technikwissenschaften e.V.: Cyber-Physical Systems - Innovationsmotor für Mobilität, Gesundheit, Energie und Produktion, acatech POSITION, Springer Verlag, Heidelberg 2011.

acatech 2015: acatech - Deutsche Akademie der Technikwissenschaften e.V.: Smart Service Welt Umsetzungsempfehlungen für das Zukunftsprojekt Internetbasierte Dienste für die Wirtschaft, Abschlussbericht Langfassung, Berlin 2015.

Beinrott 2015: Beinrott, Viktoria: Bürgerorientierte Smart City, TOGI-Schriftenreihe, Band 12, ePubli $\mathrm{GmbH}$, Berlin 2014.

Berlecon Research 2010: Berlecon Research GmbH, ZEW Mannheim, International Business School of Service Management und Pierre Audoin Consultants: Das wirtschaftliche Potenzial des Internet der Dienste; Studie im Auftrag des Bundesministerium für Wirtschaft und Technologie, Berlin 2010.

BMBF 2013: Bundesministerium für Bildung und Forschung: Zukunftsbild „Industrie 4.0“, Berlin 2013.

Chui/Löffler/Roberts 2010: Chui, Michael, Löffler, Markus und Roberts, Roger: The Internet of Things, in: The McKinsey Quarterly, 47. Jahrgang, Heft 2; Amsterdam, Atlanta 2010, S. 1 9.

Clinton 2011: Clinton, William Jefferson: Back to Work: Why We Need Smart Government for a Strong Economy, Knopf Doubleday Publishing Group, New York City 2011.

DKE 2013: Deutsche Kommission Elektrotechnik Elektronik Informationstechnik im DIN und VDE: Die deutsche Normungs-Roadmap Industrie 4.0, Reihe DKE Normungsroadmap, Version 1.0, Berlin 2013.

dpa 2015: deutsche presseagentur: Google stoppt den Verkauf seiner Datenbrille, in: Frankfurter Allgemeine Zeitung, 15. Januar 2015, Frankfurt 2015.

Ghosn 2015: Ghosn, Carlos: "Wir werden keine Taxifahrer mehr brauchen", Carlos Ghosn im Gespräch über Märkte und Motoren, Frankfurter Allgemeine Zeitung vom 11. Juli 2015, Frankfurt 2015, S. 24.

Forschungsunion/acatech 2013: Promotorengruppe Kommunikation der Forschungsunion Wirtschaft - Wissenschaft und acatech - Deutsche Akademie der Technikwissenschaften e.V.: Deutschlands Zukunft als Produktionsstandort sichern - Umsetzungsempfehlungen für das Zukunftsprojekt Industrie 4.0, Abschlussbericht des Arbeitskreises Industrie 4.0, Frankfurt 2013. 
Fromm 2015: Fromm, Jens: Verwaltung x.0, Behörden Spiegel Online - Newsletter E-Government, Informationstechnologie und Politik, Ausgabe 723, Bonn 2015, S. 7 - 8.

Gartner 2014: Gartner Inc.: Gartner Identifies the Top 10 Strategic Technology Trends for Smart Government, Pressemitteilung vom 14. April 2014, Dubai 2014.

Geiger et al. 2013: Geiger, Christian; von Lucke, Jörn; Raffl, Celina; Große, Katharina; Ramsauer, Katharina und Jandeisek, Isabel: Web 2.0 in bayerischen Kommunen, 4 Teile, Deutsche Telekom Institute for Connected Cities, Innovationsstiftung Bayerische Kommune und Zeppelin Universität gemeinnützige GmbH, Friedrichshafen/München 2013.

Geisberger/Broy 2012: Geisberger, Eva und Broy, Manfred: agendaCPS - Integrierte Forschungsagenda Cyber-Physical Systems, acatech Studie, acatech - Deutsche Akademie der Technikwissenschaften e.V., München/Garching 2012.

Habbel 2014: Habbel, Franz-Reinhard: Gastbeitrag - Vernetzt euch!, in: Willi Kaczorowski: Die smarte Stadt - Den digitalen Wandel intelligent gestalten - Handlungsfelder Herausforderungen Strategien, Richard Boorberg Verlag, Stuttgart 2014, S. 14 - 15.

Hogrebe/Kruse 2014b: Hogrebe, Frank und Kruse, Wilfried: Deutschland 4.0 - Industrie Verwaltung - Standort - Wohlstand, Grundwerk zur "Verwaltung 4.0“ als Partner von „Industrie 4.0“ im Zeitalter des Internets der Dinge und Dienste, Verlag für Verwaltungswissenschaft, Frankfurt 2014.

IJCAI 2015: International Joint Conference on Artificial Intelligence: Autonomous Weapons: an Open Letter from Al \& Robotics Researchers, International Joint Conference on Artificial Intelligence, Buenos Aires 2015.

Jiménez-Gómez et al. 2015: Jiménez-Gómez, Carlos E; Falcone, Francisco; Solanas, Agusti; Puyosa, Héctor; Zoughbi, Saleem und González, Federico: Smart Government: Opportunities and Challenges in Smart Cities Development - An IT \& Public Organization Approach, in: Ćemal Dolićanin, Ejub Kajan, Dragan Randjelović und Boban Stojanović (Hrsg.): Handbook of Research on Democratic Strategies and Citizen-Centered E-Government Services, IGI Global, Hershey 2015, S. 389 - 405.

Kaczorowski 2014: Willi Kaczorowski: Die smarte Stadt - Den digitalen Wandel intelligent gestalten Handlungsfelder Herausforderungen Strategien, Richard Boorberg Verlag, Stuttgart 2014.

Kruse/Hogrebe 2013: Kruse, Wilfried und Hogrebe, Frank: “Industrie 4.0" braucht „Verwaltung 4.0" - Globaler Wettbewerb, demographischer Wandel, Schuldenbremse, Behörden Spiegel, Berlin.

Kruse 2015: Kruse, Wilfried: Deutschland 4.0 - Der alternativlose Weg für "Made In Germany“ und die Wohlstandsgrundlage der Zukunft?! - Das Internet der Dinge und der Dienste zur "4.0 Staatsmodernisierung" in neuer Qualität nutzen!, Effizienter Staat, Berlin 2015.

von Lucke 2010: von Lucke, Jörn: Open Government - Öffnung von Staat und Verwaltung - Gutachten für die Deutsche Telekom AG zur T-City Friedrichshafen, Stand: 09.05.2010, Deutsche Telekom Institute for Connected Cities, Zeppelin University gGmbH, Friedrichshafen 2010. 
von Lucke 2012: von Lucke, Jörn: Open Government Collaboration - Offene Formen der

Zusammenarbeit beim Regieren und Verwalten - Gutachten für die Deutsche Telekom AG zur T-City Friedrichshafen, Stand: 25.10.2012, Deutsche Telekom Institute for Connected Cities, Zeppelin Universität gemeinnützige GmbH, Friedrichshafen 2012.

von Lucke 2015: von Lucke, Jörn: Technische urbane Innovationen - Potentiale von Open Government, offenen Daten und intelligenten Städten, in: Norbert Kersting (Hrsg.): Urbane Innovation, 2015, in Veröffentlichung.

von Lucke/Reinermann 2000: von Lucke, Jörn und Reinermann, Heinrich: Speyerer Definition von Electronic Government, Forschungsinstitut für öffentliche Verwaltung, Speyer 2000.

von Lucke/Schumacher 2014: von Lucke, Jörn und Schumacher, Florian: Whitepaper Verwaltung 4.0, internes Diskussionspapier E6, The Open Government Institute, Zeppelin Universität, Friedrichshafen 2014.

von Lucke/Schumacher 2015: von Lucke, Jörn und Schumacher, Florian: Erste Skizze zur Verwaltung 4.0: Neuartige Formen der Kooperation in der öffentlichen Verwaltung durch intelligente Objekte und cyberphysikalische Systeme, in: Erich Schweighofer, Franz Kummer und Walter Hötzendorfer (Hrsg.): Kooperation, Tagungsband des 18. Internationalen Rechtsinformatik Symposions, Band 309, Österreichische Computergesellschaft, Wien 2015, S. 219 - 226. ISBN: 978-385403-309-7.

Palmisano 2008: Palmisano, Samuel J.: A Smarter Planet: The Next Leadership Agenda, Council on Foreign Relations, New York 2008.

Pfirrmann 2013: Pfirmann, Oliver: Nachhaltigkeit, gute Arbeit und Innovation in der Industrie 4.0, Vortrag im Rahmen der Ringvolesung des DGB und der TU Berlin, TU Berlin, Berlin 2013.

Porter/Heppelmann 2014: Porter, Michael E. und Heppelmann, James E.: How Smart, Connected Products Are Transforming Competition, Harvard Business Review, 92. Jahrgang, Heft 11, 2014, S. $65-88$.

Raffl et al. 2014: Raffl, Celina; von Lucke, Jörn; Müller, Oliver; Zimmermann, Hans-Dieter und vom Brocke, Jan: Handbuch für offene gesellschaftliche Innovation, TOGI-Schriftenreihe, Band 11, 2. Auflage, ePubli GmbH, Berlin 2014.

Rifkin 2014: Rifkin, Jeremy: The Zero Marginal Cost Society: The Internet of Things, the Collaborative Commons, and the Eclipse of Capitalism, Palgrave Macmillan, New York 2014.

Rifkin 2014b: Rifkin, Jeremy: Digital Europe - The Rise of the Internet of Things and the Integration of the Single Market, in: European Commission: Digital Action Day 2014, Brüssel 2014.

Rubel 2012: Rubel, Thom: Smart Government - Creating More Effective Information and Services, International Data Corporation (IDC), Framingham 2012.

TSB 2014: Technologie-Stiftung Berlin: Digitales Gold - Nutzen und Wertschöpfung durch Open Data für Berlin - Daten und Fakten, TSB Technologiestiftung Berlin, Berlin 2014.

VDE-ITG 2014: Die Informationstechnische Gesellschaft im Verband der Elektrotechnik Elektronik Informationstechnik e.V.: Positionspapier „Das Taktile Internet“, Frankfurt 2014. 
Weber 2015: Weber, Mike: Verwaltung x.0, ÖFIT-Trendschau - Öffentliche Informationstechnologie in der digitalisierten Gesellschaft, Nr. 29, Fraunhofer Institut FOKUS, Berlin 2015.

Wiktionary 2011-15: Wiktionary - Das freie Wörterbuch: smart, Wikimedia Foundation Inc., San Francisco 2011-15.

WISE 2014: Warsaw Institute for Economic Studies: Big and Open Data in Europe - A growth engine or a missed opportunity?, Warsaw Institute for Economic Studies, Warschau 2014.

WSW 2011: Wiener Stadtwerke Holding: Smart City: Begriff, Charakteristika und Beispiele, Materialien der Wiener Stadtwerke zur nachhaltigen Entwicklung, Band 7, Wiener Stadtwerke Holding AG, Wien 2011. 